PHYSICAL REVIEW D 89, 123511 (2014)

\title{
Data analysis methods for testing alternative theories of gravity with LISA Pathfinder
}

\author{
Natalia Korsakova, ${ }^{1, *}$ Chris Messenger, ${ }^{2}$ Francesco Pannarale, ${ }^{3}$ Martin Hewitson, ${ }^{1}$ and Michele Armano ${ }^{4}$ \\ ${ }^{1}$ Albert-Einstein-Institut, Max-Planck-Institut für Gravitationsphysik und Universität Hannover, \\ Callinstrasse 38, 30167 Hannover, Germany \\ ${ }^{2}$ SUPA, University of Glasgow, Glasgow G12 8QQ, United Kingdom \\ ${ }^{3}$ School of Physics and Astronomy, Cardiff University, Queens Buildings, The Parade, \\ Cardiff CF24 3AA, United Kingdom \\ ${ }^{4}$ ESAC, European Space Agency, Camino bajo del Castillo s/n, Urbanización Villafranca del Castillo, \\ Villanueva de la Cañada, 28692 Madrid, Spain \\ (Received 29 April 2014; published 26 June 2014)
}

\begin{abstract}
In this paper we present a data analysis approach applicable to the potential saddle-point fly-by mission extension of LISA Pathfinder (LPF). At the peak of its sensitivity, LPF will sample the gravitational field in our Solar System with a precision of several $\mathrm{fm} / \mathrm{s}^{2} / \sqrt{\mathrm{Hz}}$ at frequencies around $1 \mathrm{mHz}$. Such an accurate accelerometer will allow us to test alternative theories of gravity that predict deviations from Newtonian dynamics in the nonrelativistic limit. As an example, we consider the case of the Tensor-Vector-Scalar $(\mathrm{TeVeS})$ theory of gravity and calculate, within the nonrelativistic limit of this theory, the signals that anomalous tidal stresses generate in LPF. We study the parameter space of these signals and divide it into two subgroups, one related to the mission parameters and the other to the theory parameters that are determined by the gravity model. We investigate how the mission parameters affect the signal detectability concluding that these parameters can be determined with the sufficient precision from the navigation of the spacecraft and fixed during our analysis. Further, we apply Bayesian parameter estimation and determine the accuracy to which the gravity theory parameters may be inferred. We evaluate the portion of parameter space that may be eliminated in case of no signal detection and estimate the detectability of signals as a function of parameter space location. We also perform a first investigation of non-Gaussian "noise glitches" that may occur in the data. The analysis we develop is universal and may be applied to anomalous tidal stress induced signals predicted by any theory of gravity.
\end{abstract}

DOI: $10.1103 /$ PhysRevD.89.123511

PACS numbers: 07.05.Kf, 04.50.Kd, 04.80.-y, 04.80.Cc

\section{INTRODUCTION}

LISA Pathfinder (LPF) [1] is a technology demonstration mission for future space-based gravitational-wave observatories, such as the Laser Interferometer Space Antenna (LISA). LPF is designed to test many of the challenging technologies needed for space-based gravitational-wave detectors and is planned to be launched in July 2015. On the basis of the LISA concept, "The Gravitational Universe" theme (with eLISA as foreseen implementation) was proposed to the European Space Agency (ESA) [2] and was selected as a science theme for the third large-class mission [3] to be launched in 2034 within the ESA Cosmic Vision science program. eLISA is a reduced version of the original LISA design that will nevertheless be able to observe numerous extremely interesting sources of gravitational waves.

LPF is a compact version of one arm of eLISA, designed to verify the ability to place test masses in free fall at the required sensitivity level. It consists of two equal test masses that are accommodated within one spacecraft.

*natalia.korsakova@aei.mpg.de
The instrument measures the relative position of two freefalling test masses with picometer precision using laser interferometry, thus being sensitive to the differential gradients of the gravitational potential. LPF will initially be placed in a Lissajous orbit around L1, the Lagrangian point of dynamically unstable equilibrium between the Sun and the Earth, where the gravitational forces and the centrifugal force cancel out in the noninertial rotating reference frame. The transition from Earth to L1 will take three months and will be followed by six months of experiments performed to verify the on-board technologies and performance of the satellite [4]. It was noted [5,6] that the combination of design solutions for the mission, such as the sampling frequency and the overall measurement sensitivity, would allow LPF to probe anomalous gravity stress tensors, i.e. ones that deviate from the Newtonian prediction, in the low gravity regime. Anomalous stress tensors are predicted by various alternative theories of gravity and high precision measurements of these deviations would allow us to test such theories. To this end, ESA scientists and members of the science and industrial community have been studying a possible LPF mission extension. Here we consider the data analysis methods for such a scenario. 
In the Solar System, the low gravity regime can be investigated at the saddle points (SPs) of two-body systems, where the gradients of the gravitational potential of two gravitating bodies are equal in magnitude and opposite in orientation. For the Sun-Earth system, the SP is located about $1231000 \mathrm{~km}$ away from L1 towards Earth. A SP is not an equilibrium point, so it will only be possible to perform a "fly-by" with LPF. When passing by the SP, LPF will be sampling the gravity stress tensor in a low gravitygradient region. The measured variation of the distance between the two test masses can be compared to the theoretical predictions from Newtonian and alternative theories of gravity. From these comparisons one can infer (i) if any deviations from Newtonian dynamics occur, and (ii) constrain alternative theories of gravity.

The data analysis approach developed in this paper allows for a rigorous analysis of the test made during the SP fly-by. It aims at exploring the possible deviations from the Newtonian dynamics by analyzing the gravity stress tensor measured by LPF.

We consider the class of alternative theories of gravity that have modified Newtonian dynamics (MOND) in their nonrelativistic limit. MOND emerged as a possible way to explain the observations of rotational curves of spiral galaxies [7-12]. The observations show that the rotational curves of the galaxies stay constant and do not depend on the distance from the galactic center, as expected in Newtonian gravity. MOND (originally proposed by Milgrom [13]) is a possible heuristic solution to this problem, in contrast to the introduction of hidden mass (i.e. dark matter). At the core of the theory is a characteristic acceleration $a_{0} \approx 10^{-10} \mathrm{~m} / \mathrm{s}^{2}$ at which a transition occurs, from the regime accurately described by the Newtonian field equation, to one in which the gravitational dynamics is better described by a nonlinear Poisson equation. To embed MOND into a consistent theory of gravity, we chose TensorVector-Scalar $(\mathrm{T} e \mathrm{~V} e \mathrm{~S})$ as underpinning relativistic theory, bearing in mind that other choices could be possible. The key details are presented in Sec. IV B together with the rationale behind our choice.

Generally speaking, alternative theories of gravity that incorporate MOND as an additional scalar field can all be parametrized in the same way. In addition to the function that describes the transition from the MONDian to the Newtonian regime, the contribution of the additional scalar gravity potential introduced by these theories to the overall physical potential will depend on two parameters. The first parameter is also inherited from initial MOND heuristics and stands for the characteristic acceleration $a_{0}$ mentioned earlier. The other parameter determines the coupling of the additional scalar field to the overall physical potential. In this respect, the analysis that is going to be performed here for the TeVeS theory can be easily extended to the entire class of similar theories.
In order to study the detection of a signal of a particular shape in additive noise, as in the LPF SP fly-by scenario, one must first determine the physical quantities that influence the form of the signal itself. In our case, we parametrize the signal in terms of two groups of physical quantities. The first set of parameters is determined by the way the stress tensor is sensed by the instrument and will depend on the fly-by trajectory and the orientation of the LPF sensitive axis joining the two free-falling test masses. The second set of parameters is prescribed by the theory of gravity that determines the anomalous stress tensor under consideration and varies from theory to theory. The parameters that come from the experiment setup, or mission parameters, can be estimated during the flight independently of the main scientific measurement. The position of the spacecraft in space as a function of time will be determined using standard spacecraft tracking techniques, and its orientation will be measured using on-board star trackers. One of our goals is to determine whether and how much the accuracy of these measurements will influence our ability to detect a deviation from Newtonian gravity. With this objective in mind, we quantify how mission parameters variations will influence the measured signal and how much this differs from the true signal, modeled using fixed values obtained from other observations.

Primarily, we want to measure (or constrain) the second group of parameters with LPF and, in case of no signal detection, to draw conclusions about the validity of a specific theory of gravity under consideration. We chose to use a Bayesian approach to estimate the parameter values. Further, we apply Bayes' theorem to address the problem of model selection, in which we must choose between two models, one that predicts the presence of a signal in the data and the other that assumes the data to be noise only. For the analysis of the theory parameters, the simulated data is constructed by summing Gaussian noise, with a known amplitude spectral density, and an anomalous tidal stress signal. We construct simulated signals by solving the MOND nonlinear Poisson equation [see Eq. (19)] numerically (with the help of the code provided by our colleagues from Imperial College London [14]) in a neighborhood of the Sun-Earth SP and by simulating the passage of LPF along a given satellite trajectory and with a fixed tidal stress sampling rate. We show the parameter estimation results for several representative points in the parameter space. We also show the outcome of the noiseonly scenario and determine the area of the parameter space that will be ruled out in case of no signal detection. Furthermore, we present model selection results for several points in the parameter space. Finally, we apply the data analysis framework to realistic data from an LPF test campaign and discuss both parameter estimation and model selection results. This data set is interesting as it contains a noise artifact that can be misinterpreted by the data analysis setup as a signal. 
An important remark regarding the example of applying our data analysis framework to the MOND limit of TeVeS must be made. Tests for alternative theories of gravity, including $\mathrm{Te} e \mathrm{~V}$, are performed in the strong field regime by measuring the orbital decay of the relativistic pulsarwhite dwarf binary PSR J1738 + 0333 [15]. The constraints imposed to the theory in its strong field limit, however, differ from the ones that can be imposed in the weak field limit [16]. The constraints that would follow from the method described in this paper would therefore be complementary to, say, the PSR J1738+0333 ones and largely applicable to theories exhibiting the same scalar field coupling mechanism as $\mathrm{TeVeS}$.

The paper is structured as follows. In Sec. II we discuss LPF and explain how it performs measurements. In Sec. III we identify the mission parameters and discuss how the trajectory of the spacecraft and the projection of the signal on the LPF sensitive axis will influence the signal. Section IVA describes the two approaches we develop for the analysis framework of the mission and theory parameters. In Sec. IV B, in order to fix an example against which our data analysis tools may be tested, we briefly describe the nonrelativistic limit of $\mathrm{TeVeS}$ theory of gravity, and we report on the signal model construction and the space of theory parameters for this scenario. We present our results in Sec. V and gather our conclusions in Sec. VI, where we also discuss possibilities of future work for this experiment.

\section{LISA PATHFINDER}

The task of measuring the residual differential acceleration of two free-falling test masses is one of the main objectives of the LPF mission and, therefore, the conversion from the observed differential displacements to differential accelerations has been analyzed in depth $[17,18]$.

\section{A. LPF measurement}

LPF measures differential displacements between two free-falling test masses and is thus sensitive to their differential acceleration [17]. Consider the relative motion of two masses that follow the geodesics of the gravitational field and let the vector $\zeta$ denote the separation between the two test masses. The components of this vector may be expressed as $\zeta^{i}=x_{1}^{i}-x_{2}^{i}$, where $x_{\{1,2\}}^{i}$ are the coordinates of the two test masses. Working in Cartesian coordinates, the equations of motion for the test masses in Newtonian gravity are

$$
\frac{\mathrm{d}^{2} x_{1}^{i}}{\mathrm{~d} t^{2}}=-\frac{\partial \Phi_{\mathrm{N}}\left(\boldsymbol{x}_{1}, t\right)}{\partial x^{i}}
$$

and

$$
\frac{\mathrm{d}^{2} x_{2}^{i}}{\mathrm{~d} t^{2}}=-\frac{\partial \Phi_{\mathrm{N}}\left(\boldsymbol{x}_{2}, t\right)}{\partial x^{i}},
$$

where $\Phi^{\mathrm{N}}$ is the Newtonian gravitational potential. The relative acceleration is thus given by

$$
\begin{aligned}
\frac{\mathrm{d}^{2} \zeta^{i}}{\mathrm{~d} t^{2}} & =\frac{\mathrm{d}^{2} x_{1}^{i}}{\mathrm{~d} t^{2}}-\frac{\mathrm{d}^{2} x_{2}^{i}}{\mathrm{~d} t^{2}} \\
& =-\zeta^{j} \frac{\partial^{2} \Phi_{\mathrm{N}}}{\partial x^{i} \partial x^{j}}+o\left(\zeta^{i} \zeta_{i}\right)=-\mathcal{E}^{i}{ }_{j} \zeta^{j}+o\left(\zeta^{i} \zeta_{i}\right),
\end{aligned}
$$

where summation over repeated indices is implied, the gravitational potential is expanded in terms of the separation vector up to the first order, and $\mathcal{E}_{i j}=\partial^{2} \Phi_{\mathrm{N}} / \partial x^{i} \partial x^{j}$ is the gravitational tidal field in Cartesian coordinates [19].

LPF has one sensitive axis that is oriented along the line joining the two free-falling test masses. By projecting Eq. (3) along this axis, one obtains

$$
\frac{\mathrm{d}^{2} \zeta_{i}}{\mathrm{~d} t^{2}} \hat{\zeta}^{i}=-\hat{\zeta}^{i} \zeta^{j} \frac{\partial^{2} \Phi_{\mathrm{N}}(\vec{x}, t)}{\partial x^{i} \partial x^{j}}
$$

where $\hat{\zeta}^{i}=\zeta^{i} /\|\zeta\|$ is the $i$ th component of the unit vector in the $\zeta$ direction. The diagonal components of $\mathcal{E}_{i j}$ contribute to the relative acceleration of the test masses, whereas the remaining components contribute to their tilts. The diagonal components of the stress tensors are larger than the nondiagonal ones, therefore we will consider only the relative acceleration contribution.

\section{B. Estimation of the differential test mass acceleration}

LPF is designed to keep the distance between the two test masses constant below $1 \mathrm{mHz}$ by accounting for external forces, whereas at the sensitivity frequencies of $1-30 \mathrm{mHz}$ the test masses are in free fall. Both test masses are accommodated within one spacecraft and free fall is achieved by controlling the position of the spacecraft relative to one test mass. The position of the second test mass is then controlled relative to the first outside the LPF sensitive frequency band. The differential gravitational force can thus be recovered from the measurement of the differential displacement. An anomalous stress tensor predicted by an alternative theory of gravity may therefore be sensed by LPF as the differential force acting on the test masses. This is performed by taking into account the models $[17,18]$ of the LPF subsystems in the equations of motion for the test masses along the sensitive axis, described by

$$
\boldsymbol{a}=\left[\boldsymbol{D}^{-1} \boldsymbol{I}^{-1}+\boldsymbol{C}\right] \boldsymbol{o},
$$

where $\boldsymbol{o}=\left(o_{1}, o_{\Delta}\right)^{\mathrm{T}}$ is read interferometrically along the sensitive axis of LPF by the two interferometers on board, $o_{1}$ being the position of the first test mass relative to the spacecraft, and $o_{\Delta}$ being the position of the second test mass 


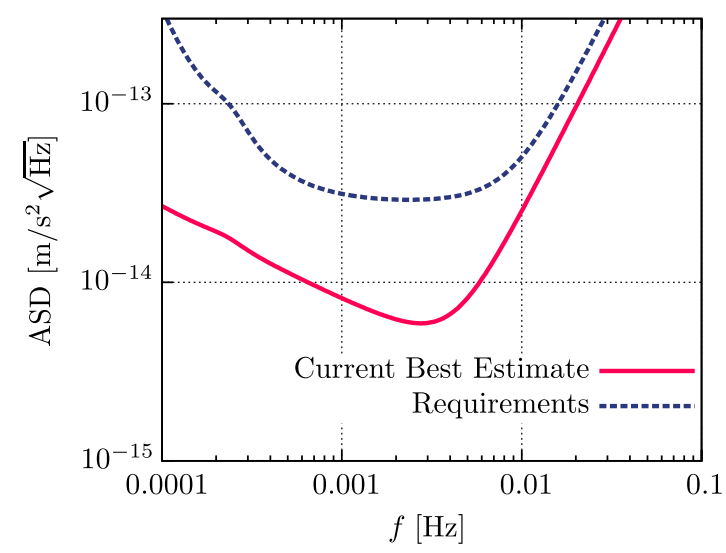

FIG. 1 (color online). LPF sensitivity. Amplitude spectral densities of the requirements and the current best noise estimates.

relative to the first. $\boldsymbol{a}=\left(a_{1}, a_{\Delta}\right)^{\mathrm{T}}$, with $a_{1}=\mathrm{d}^{2} x_{1} / \mathrm{d} t^{2}$ and $a_{\Delta}=\mathrm{d}^{2} \zeta / \mathrm{d} t^{2}$ being the estimated residual acceleration of the spacecraft and the estimated residual differential acceleration of the two test masses, respectively. $\boldsymbol{D}$ represents the dynamics of the spacecraft, $\boldsymbol{I}$ the interferometer sensing matrix, and $\boldsymbol{C}$ the controller transfer functions. More specifically, the dynamics of the spacecraft is

$$
\boldsymbol{D}=\left[\begin{array}{cc}
\frac{1}{\left(s^{2}+\omega_{1}^{2}\right)} & 0 \\
-\frac{\left(\omega_{2}-\omega_{1}\right)^{2}}{\left(s^{2}+\omega_{1}^{2}\right)\left(s^{2}+\omega_{2}^{2}\right)} & \frac{1}{\left(s^{2}+\omega_{2}^{2}\right)}
\end{array}\right],
$$

where $s$ is a Laplace domain complex variable and $\omega_{\{1,2\}}^{2}=k_{\{1,2\}} / m$. The mass of the test mass is $m$ and $k_{\{1,2\}}$ are the spring constants that model the gravitational and electrostatic couplings between the test masses and the spacecraft. Given the coupling factor $\delta$ modeling the degree to which the differential interferometer picks up motion of the spacecraft, the interferometer sensing matrix can be written as

$$
\boldsymbol{I}=\left[\begin{array}{ll}
1 & 0 \\
\delta & 1
\end{array}\right]
$$

Finally, the controller matrix that converts the measured signal into the commanded forces may be written as

$$
\boldsymbol{C}=\left[\begin{array}{cc}
H_{\mathrm{df}} & 0 \\
0 & H_{\text {sus }}
\end{array}\right]
$$

where $H_{\mathrm{df}}$ and $H_{\text {sus }}$ are the gains of the drag-free and suspension control loops along the sensitive axis of LPF, respectively. The drag-free control loop actuates on the spacecraft via micro-Newton thrusters, while the suspension loop actuates on the second test mass by electrostatic actuation.

\section{Noise sources in the LPF measurement}

LPF measurements are contaminated by the system noise. The design of LPF is such that the sensitivity of the instrument is expected to be limited by the interferometer shot noise at high frequencies and by force noise on the test masses at low frequencies. Various tests of the flight hardware, however, show that the real sensitivity of LPF is expected to exceed the design requirements [1], as shown in Fig. 1. The noise current best estimate for LPF is limited by the electrostatic actuation noise on the second test mass at low frequencies.

\section{IDENTIFICATION OF MISSION PARAMETERS}

In order to parametrize the signals measured by LPF, we must begin by defining a method to determine the spacecraft trajectory uniquely. Let us fix a right-handed Cartesian coordinate system with its origin in the Sun-Earth SP, its $x$ axis aligned with the line connecting the Earth and the Sun, and its $z$ axis perpendicular to the ecliptic (see Fig. 2). The trajectory of LPF in the neighborhood of the SP can be approximated as a straight line. The direction of the trajectory will be determined by two angles: $\eta$, the angle between the $z$ axis and the direction of the spacecraft velocity, and $\varphi$, the angle between the $x$ axis and the projection of the velocity vector on the ecliptic. The unit vector along the trajectory of the spacecraft in the direction of motion is, therefore,

$$
\left(\hat{e}_{x}, \hat{e}_{y}, \hat{e}_{z}\right)=(\sin \eta \cos \varphi, \sin \eta \sin \varphi, \cos \eta) .
$$

The point of the closest approach of the trajectory to the SP, $\left(\xi_{x}, \xi_{y}, \xi_{z}\right)$, determines the impact parameter, i.e. the distance of the fly-by, which is the length of the perpendicular dropped from the SP on the trajectory. The position of the spacecraft may thus be written as

$$
(x, y, z)=\left(\xi_{x}, \xi_{y}, \xi_{z}\right)+\left(\hat{e}_{x}, \hat{e}_{y}, \hat{e}_{z}\right) r
$$

where $r$ is the distance from the point of closest approach.

Given the distance to the saddle point, the position of the closest approach becomes redundant. Therefore, to avoid the uncertainty the two angles $\eta_{\perp}$ and $\varphi_{\perp}$ that define the position of the perpendicular to the trajectory are introduced:

$\left(\xi_{x}, \xi_{y}, \xi_{z}\right)=\|\xi\|\left(\sin \eta_{\perp} \cos \varphi_{\perp}, \sin \eta_{\perp} \sin \varphi_{\perp}, \cos \eta_{\perp}\right)$,

where $\|\xi\|$ is the length of the vector $\left(\xi_{x}, \xi_{y}, \xi_{z}\right)$. Similarly to the $(\eta, \varphi)$ notation previously introduced, $\eta_{\perp}$ denotes the angle between the perpendicular and the ecliptic, while $\varphi_{\perp}$ denotes the angle between the $x$ axis and the projection of the perpendicular on the ecliptic. Notice that the additional condition, 


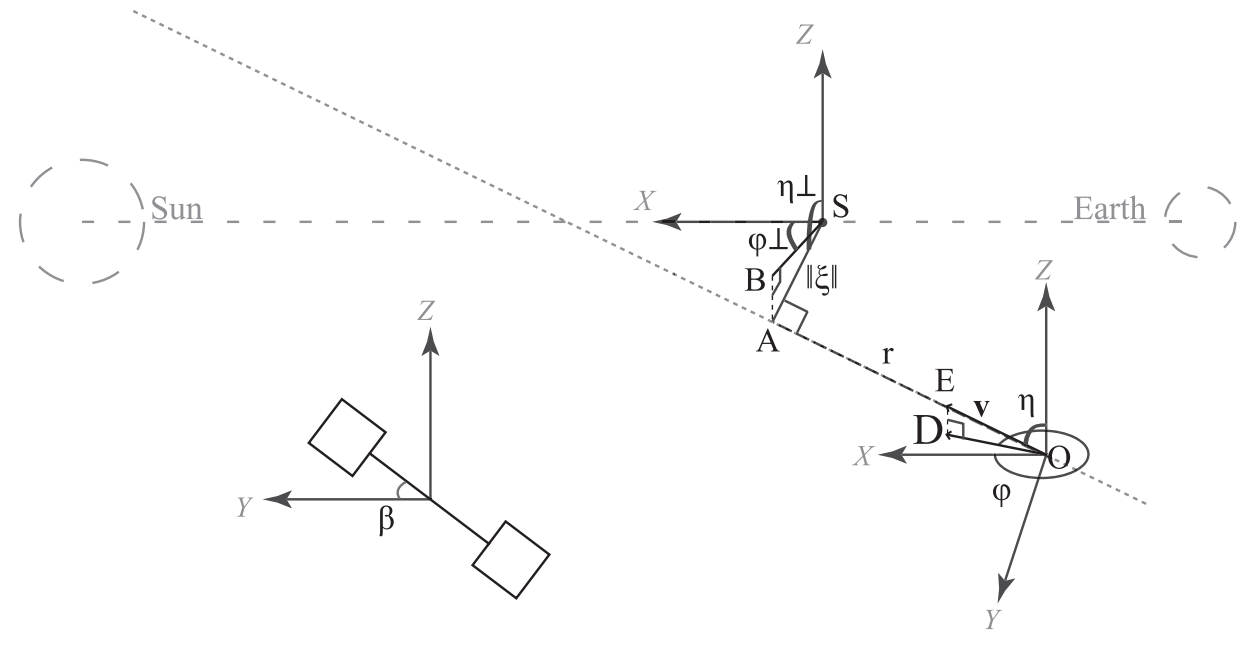

FIG. 2. Schematic of the trajectory parameters. The coordinate system has its origin in the SP (S) and the $x$ axis is parallel to the line joining the Sun and the Earth and pointing in the direction of the Sun. The $z$ axis is perpendicular to the ecliptic. The spacecraft velocity $v$ is aligned with the trajectory and represented by the vector $O E$. The direction of the trajectory is defined by the two angles $\eta$ (the angle between the $z$ axis and $v$ ) and $\varphi$ [the angle between $x$ axis and projection of $v$ on the $(x, y)$ plane, shown as the segment OD]. The position of the spacecraft along the trajectory is determined by the variable $r$, the distance to the point A where the perpendicular dropped on the trajectory intersects with it. The length of the perpendicular is given by the parameter $\|\xi\|$. The position of the perpendicular is given by two angles, $\eta_{\perp}$ (the angle between the $z$ axis and the perpendicular) and $\varphi_{\perp}$ [the angle between the $x$ axis and the projection of the perpendicular on the $(x, y)$ plane, segment BS]. The angle $\beta$ determines the projection of the gravity stress tensor on the sensitive axis of LPF.

$\sin \eta \sin \eta_{\perp}\left(\cos \varphi \cos \varphi_{\perp}+\sin \varphi \sin \varphi_{\perp}\right)+\cos \eta \cos \eta_{\perp}=0$,

holds for the four angles $\eta, \varphi, \eta_{\perp}$, and $\varphi_{\perp}$ as a consequence of the orthogonality between the satellite trajectory and the line of closest approach. This allows us to further reduce the parameters that determine the perpendicular to the trajectory of the satellite in the neighborhood of the SP down to $\eta_{\perp}$ and $\operatorname{sign}\left(\sin \varphi_{\perp}\right)$. The latter determines whether $\varphi_{\perp} \in$ $(0, \pi)$ or $\varphi_{\perp} \in(\pi, 2 \pi)$.

The signal measured by LPF can be simulated by sampling the stress tensor along the trajectory with velocity $v$ and the instrument sampling frequency of $10 \mathrm{~Hz}$. The velocity of the spacecraft and the sampling frequency determine the resolution at which the gravity stress tensor is being sampled.

As a final step, we must define the projection of the stress tensor on the sensitive axis of LPF. The projection is determined by the two angles $\alpha$ and $\beta$ that the sensitive axis forms with the $x$ axis and the $y$ axis of the coordinate system, respectively. However, since LPF is held oriented so that its solar panel faces the Sun, and since we are considering a neighborhood of the Sun-Earth SP, and because the sensitive axis of LPF is parallel to the solar panel, $\alpha$ can be fixed to $\alpha=90^{\circ}$. The projection of the stress tensor on the sensitive axis is thus determined only by the angle $\beta$, making LPF sensitive to the linear combination of the two diagonal components of the stress tensor:

$$
\frac{\mathrm{d}^{2} \zeta_{i}}{\mathrm{~d} t^{2}} \hat{\zeta}^{i}=-\|\zeta\|\left[\mathcal{E}_{y y} \cos ^{2}(\beta)+\mathcal{E}_{z z} \sin ^{2}(\beta)\right] .
$$

All in all, the signal can be fully described in terms of the following set of mission parameters:

$$
\lambda_{0}^{m}=\left\{\|\xi\|, \eta, \varphi, \eta_{\perp}, \operatorname{sign}\left(\sin \varphi_{\perp}\right),\|v\|, \beta\right\},
$$

which are depicted in Fig. 2.

\section{DATA ANALYSIS}

We now introduce the approach to the analysis of the data that will be acquired with LPF in the vicinity of the Sun-Earth SP. We describe the model of the data and the derivation of a matched filter which will be designed to study the mission parameters. Thereafter, we develop a Bayesian approach to the analysis of the theory parameters.

\section{A. Data model}

The detector noise is modeled as having a frequency dependent spectrum (see Fig. 1), hence it is more natural to carry out the analysis in the frequency domain. We write the measured data as

$$
\tilde{x}\left(f, \lambda_{0}\right)=\tilde{h}\left(f, \lambda_{0}^{m}, \lambda_{0}^{t}\right)+\tilde{n}(f),
$$

where $\tilde{h}\left(f, \lambda_{0}^{m}, \lambda_{0}^{t}\right)$ and $\tilde{n}(f)$ are the Fourier transforms of the signal and the detector noise, respectively. $\vec{\lambda}_{0}=\left(\lambda_{0}^{m}, \lambda_{0}^{t}\right)$, where $\lambda_{0}^{m}$ and $\lambda_{0}^{t}$ denote the mission and the theory parameters that govern the signal: the former are listed in Eq. (14), whereas the latter will be discussed in the course of the paper. We model the noise as Gaussian, with zero mean and two-sided noise power spectral density 


$$
S(f) \approx|\tilde{n}(f)|^{2} / \Delta f,
$$

where $\Delta f=1 / T$ is the size of the frequency bin, whereas $T=N \cdot \Delta t$ with $N$ the number of samples over the measurement time interval $[0, T]$ and $\Delta t$ the time domain sampling interval. The Fourier transform of the noise averaged over ensemble is the variance of the noise $\sigma^{2}=\left\langle|\tilde{n}(f)|^{2}\right\rangle$. The noise models we use are defined by the theoretical amplitude spectral density (ASD) shown in Fig. 1.

In order to test our data analysis framework on artificial data, we must choose a model to produce signal templates. As anticipated in the Introduction, in this paper we consider the stress tensor predictions obtained within the nonrelativistic limit of Bekenstein's TeVeS theory of gravity. This theory embeds the heuristic description of the dynamics of galaxies provided by MOND into a consistent relativistic theory (see Appendix A).

\section{B. Building signal templates}

\section{Nonrelativistic limit of $\mathrm{TeV} e \mathrm{~S}$}

As we are going to perform the experiment in the Solar System, we must consider the quasistatic, weak potential, and slow motion limit of $\mathrm{Te} e \mathrm{~S}$ [20]. We may thus take the metric to be time independent. Additionally, as we work in a neighborhood of the Sun-Earth SP, far enough from both bodies, we may set the metric to be flat. In the nonrelativistic limit, the full physical potential that determines the test particle acceleration within $\mathrm{T} e \mathrm{~V} e \mathrm{~S}, \vec{a}=-\nabla \Phi$, is given by the sum of the Newtonian vector potential $\Phi_{\mathrm{N}}$ and the scalar potential $\phi$, i.e.

$$
\Phi=\Phi_{\mathrm{N}}+\phi+\mathcal{O}\left(\Phi_{\mathrm{N}}^{2}\right) .
$$

Therefore LPF will be measuring $\Phi$, which has to replace $\Phi_{\mathrm{N}}$ in Eqs. (1)-(4). The Newtonian potential is given by the familiar Poisson equation

$$
\nabla^{2} \Phi_{\mathrm{N}}=4 \pi G \tilde{\rho},
$$

where $\tilde{\rho}$ is the baryonic mass density, whereas the scalar potential $\phi$ is determined by the nonlinear Poisson equation

$$
\nabla \cdot\left[\mu\left(k l^{2}(\nabla \phi)^{2}\right) \nabla \phi\right]=k G \tilde{\rho},
$$

where $k$ is a dimensionless constant and $l$ is a constant length.

The $\mu$ function appearing in the last equation is a free function that governs the transition from the Newtonian regime to the MONDian one [see Eq. (A3)]. We can reparametrize its dimensionless argument $y \equiv k l^{2}(\nabla \phi)^{2}$ in terms of an acceleration parameter

$$
a_{0} \equiv \frac{(3 k)^{1 / 2}}{4 \pi l},
$$

thus obtaining

$$
y=3\left(\frac{k}{4 \pi}\right)^{2}\left(\frac{\nabla \phi}{a_{0}}\right)^{2},
$$

where the ratio between the MONDian acceleration and the acceleration parameter is now manifest. The asymptotical limits of the $\mu$ function must therefore obey the following requirements:

$$
\begin{array}{ll}
\mu(y) \rightarrow 1, & \text { for } y \rightarrow \infty, \\
\mu(y) \approx \sqrt{y / 3}, & \text { for } y \ll 1,
\end{array}
$$

where the first condition leads to the Newtonian regime. The second condition ensures that in the low acceleration regime, i.e. $|\nabla \Phi| \ll a_{0}$, the MOND modification originally proposed by Milgrom [13] generates a different dynamics, recovering, for example, the one exhibited by rotational curves of galaxies.

\section{Signal model and parameter space}

As shown by Eqs. (19)-(21), within the example selected for this paper, the signal models will be determined by two parameters $k$ and $a_{0}$, and a free function, $\mu$. For the moment, we fix the $\mu$ function to the form that was proposed in [20]. In terms of the notation introduced in Eq. (15), therefore, $\lambda_{0}^{t}=\left\{k, a_{0}\right\}$. The nonlinear elliptical differential equation which determines the scalar potential $\phi$ and hence the tidal stress tensor, Eq. (19), can be solved numerically [14] (the code that implements the numerical solution was kindly provided by Imperial College London). While in [20] the $\mu$-function definition is

$$
y=\frac{3}{4} \frac{\mu^{2}(\mu-2)^{2}}{1-\mu},
$$

the interpolating function $\mu$ in our numerical calculations is fixed via the relation

$$
\frac{\hat{\mu}}{\sqrt{1-\hat{\mu}^{4}}}=\frac{k}{4 \pi} \frac{|\nabla \phi|}{a_{0}},
$$

where we used the notation $\hat{\mu}$ to explicitly distinguish this function from the one appearing in Eq. (23). As shown in Fig. 3, the two functions are in a good agreement. The advantage of $\hat{\mu}$ is that it may be written out analytically as

$$
\hat{\mu}=\sqrt{\frac{-1+\sqrt{1+4 x^{2}}}{2 x}},
$$

where $x=y / 3$. In solving the nonlinear Poisson equation numerically, the condition $\mu=\sqrt{x}$ for $x<10^{-5}$ is used [see Eq. (22)]. 


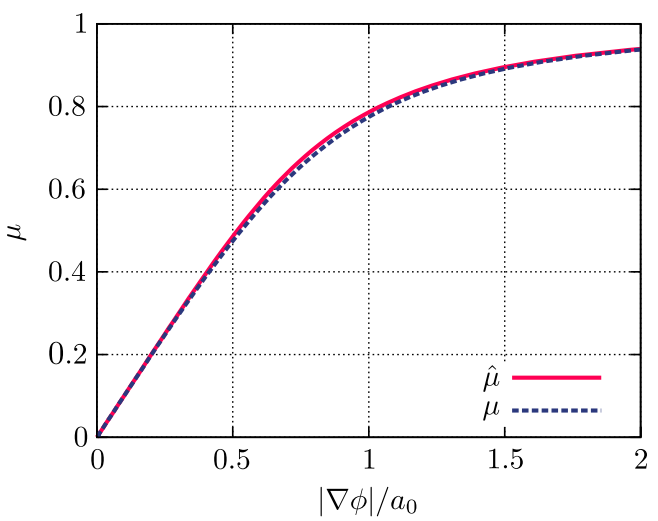

FIG. 3 (color online). Comparison between the interpolating function used for the numerical calculations and the one originally proposed in [20].

To solve Eq. (19) numerically, other than fixing the $\mu$ function, we must prescribe boundary conditions. We use the rescaled Newtonian potential for this purpose. This is readily obtained from Eqs. (18) and (19) by taking into account that $\mu \rightarrow 1$ as $|\nabla \phi| / a_{o} \rightarrow \infty$ and by applying Gauss's theorem. This yields

$$
\nabla \phi=\frac{k}{4 \pi} \nabla \Phi_{\mathrm{N}},
$$

so that the gradient of the physical potential $\Phi$ reduces to the usual Newtonian form with a renormalized gravitational constant given by

$$
G_{\mathrm{N}}=\left(1+\frac{k}{4 \pi}\right) G
$$

In order to produce signal templates for LPF, as a first step we compute the spatial derivatives of $\nabla \phi$ at each grid point. This provides the nine stress tensor components, namely, $\partial^{2} \phi / \partial x_{i} \partial x_{j}$, where $x_{i, j}=x, y, z$, at each point of the lattice. Once this is done, we must prescribe values for the set of mission parameters listed in Eq. (14) and sample the stress tensor along the LPF trajectory [Eq. (10)]. The sampling points are determined by the spacings $\|(\Delta x, \Delta y, \Delta z)\|=\|v\| \Delta t$, with time step $\Delta t=1 / f_{\text {samp},}$ $f_{\text {samp }}=10 \mathrm{~Hz}$ being the LPF sampling frequency. The stress tensor components are calculated at each sampling point by performing a trilinear interpolation on a threedimensional irregular grid. The interpolation procedure starts with a linear interpolation in the $x$-axis direction. This is followed by a linear interpolation along the $y$ axis employing the $x$-interpolated values. Finally, both the $x$ - and $y$-interpolated values are used to perform the linear interpolation in the $z$ direction.

Our goals are (1) to see how the signal templates change when varying the two theory parameters $k$ and $a_{0}$, and (2) to study their detectability in the noise. The value of the dimensionless coupling constant $k$ should be of the order $10^{-2}$ to be consistent with the cosmological expansion; $k=$ 0.03 is chosen in [20]. The characteristic acceleration is usually set to $a_{0} \approx 10^{-10} \mathrm{~m} / \mathrm{s}^{2}$, in accordance with observations of rotational curves of galaxies [21]. We vary both parameters within reasonable ranges around their "original" values, so that $k \in[0 ; 0.12]$ and $a_{0} \in\left[0 ; 4 \times 10^{-10}\right] \mathrm{m} / \mathrm{s}^{2}$. We cover this two-dimensional space of theory parameters with a $9 \times 9$ uniform grid (see Fig. 14) and solve Eq. (19) numerically in the neighborhood of the Sun-Earth SP for all choices of $\left(k, a_{0}\right){ }^{1}$ We then fix a set of trajectory parameters and produce LPF signal templates by projecting the computed stress tensor as in Eq. (13), at all points in the $\left(k, a_{0}\right)$ parameter space. Additionally, we set $\partial \phi^{2} / \partial x_{i} \partial x_{j}=0$ along $k=0$ and $a_{0}=0 \mathrm{~m} / \mathrm{s}^{2}$, as proposed in [20]. In order to obtain signal templates for generic values of $k$ and $a_{0}$, we use a bicubic interpolation along both directions. We interpolate the signal templates from the knows solutions for the stress tensor on the two-dimensional parameter space. The interpolation is performed for each sample in the template time series. This is possible since, for a given set of trajectory parameters, a sample in the template time series represents the same position in time and in space for a particular choice of $a_{0}$ and $k$.

As a final remark, we note that in some instances the choice of the theory parameters requires to extend the templates outside the lattice where the MONDian stress tensor is calculated. As this extension must be performed in a Newtonian limit regime, we exploit the scaling relation between the Newtonian stress tensor (analytically computed, see Appendix B) and the MONDian one: these are related by a factor $k / 4 \pi$ [see Eqs. (26) and (27)], so that projecting the rescaled Newtonian stress tensor along the LPF sensitive axis allows us to extend the MONDian template. An example of this is shown in Fig. 4.

\section{Analysis of the mission parameters}

In this section we study how the template of the predicted signal changes when varying the mission parameters. This knowledge will validate our choice in studying the theory and the mission parameters independently. This greatly simplifies the study of theories that predict signals that can be measured with LPF. To investigate the mission parameter space we fix the theory parameters to $k=0.03$ and $a_{0}=10^{-10} \mathrm{~m} / \mathrm{s}^{2}$, following [20]. In this section, for the sake of simplicity, we also remove references to the theory parameters from the notation.

We begin by introducing the concept of a linear filter. In terms of our problem, it is a signal template with a certain set of parameters. Its construction is based on the "true" signal that has a fixed set of (mission) parameters $\lambda_{0}^{m}$. In order to quantitatively assess the influence of parameter variations, we estimate the response of the filter to "data"

\footnotetext{
${ }^{1}$ Calculations were performed using [22].
} 


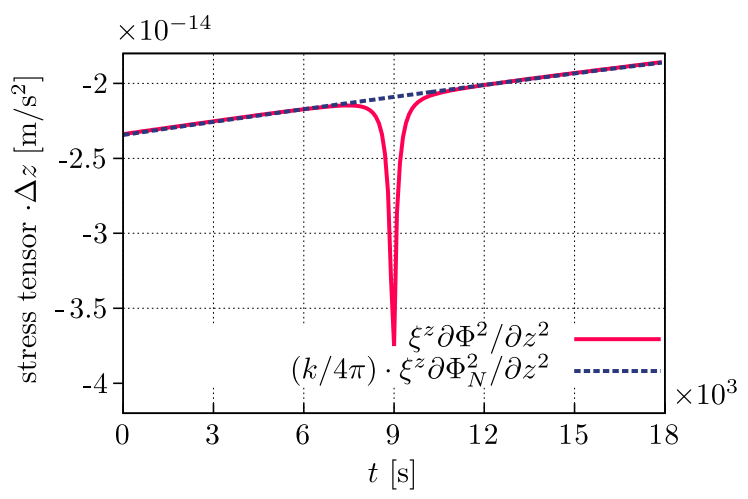

FIG. 4 (color online). Comparison between a template produced with a numerical calculation and the rescaled Newtonian background analytically estimated using Eq. (B6). In this example, $k=0.03$ and $a_{0}=10^{-10} \mathrm{~m} / \mathrm{s}^{2}$. The $\partial \Phi^{2} / \partial z^{2}$ and $\partial \Phi_{N}^{2} / \partial z^{2}$ components of the MONDian and Newtonian stress tensors are plotted. This means that the sensitive axis is parallel to the $z$ axis of the coordinate system and, therefore, that $\beta=0$.

generated using mission parameters $\lambda_{\mathrm{v}}^{m}$ that have an offset $\Delta \lambda^{m}=\lambda_{\mathrm{v}}^{m}-\lambda_{0}^{m}$ within the range of spacecraft navigation errors reported in Table I. This table provides the accuracy with which each parameter can be determined from navigation system measurements. We report both the errors on the mission parameters assigned before the flight (Uncertainty before the flight) and the precision attainable during the flight by spacecraft navigation system measurements (Uncertainty after the flight) $[23,24]$. Notice that the low precision on the angles $\varphi$ and $\eta$ before the flight follows from the uncertainty on the trajectory which depends on the departure conditions from the Lissajous orbit around L1 [23] and they will be known better once the trajectory is chosen.
The correlation between the data, $\tilde{x}$, and a signal template, $\tilde{q}$, can be calculated as the output of a matched filter via

$$
C\left(\tau, \Delta \lambda^{m}\right)=\int_{-\infty}^{\infty} \tilde{x}\left(f, \lambda_{\mathrm{v}}^{m}\right) \tilde{q}^{*}\left(f, \lambda_{0}^{m}\right) e^{-2 \pi i f \tau} \mathrm{d} f .
$$

The signal at the output of the matched filter is the averaged correlation function, for which $\left\langle\tilde{x}\left(f, \lambda_{\mathrm{v}}^{m}\right)\right\rangle=\left\langle\tilde{h}\left(f, \lambda_{\mathrm{v}}^{m}\right)\right\rangle$ since $\langle\tilde{n}(f)\rangle=0$. We do not take into account the time delay $\tau$ of the signal arrival. We assume that the expected time of the signal arrival, which is the time when the spacecraft has its closest approach to the SP, is known. The error on the time of the signal arrival is embedded in the parameter that defines the distance from the SP to the point where the measurement is made. The mean of the correlation function between the data on the output of the instrument and the linear filter $\tilde{q}$ [26] thus reads

$$
\hat{C}\left(\Delta \lambda^{m}\right)=\int_{-\infty}^{\infty} \tilde{h}\left(f, \lambda_{\mathrm{v}}^{m}\right) \tilde{q}^{*}\left(f, \lambda_{0}^{m}\right) \mathrm{d} f
$$

By setting the linear filter to the true template weighted by the noise power spectral density, i.e.

$$
\tilde{q}^{*}\left(f, \lambda_{0}^{m}\right)=\frac{\tilde{h}^{*}\left(f, \lambda_{0}^{m}\right)}{S(f)},
$$

the filter becomes optimal [27]. An optimal matched filter is one that maximizes the (SNR)

$$
\rho^{2}=\hat{C}\left(\Delta \lambda^{m}=0\right)=\int_{-\infty}^{\infty} \frac{\tilde{h}\left(f, \lambda_{0}^{m}\right) \tilde{h}^{*}\left(f, \lambda_{0}^{m}\right)}{S(f)} \mathrm{d} f
$$

TABLE I. This table lists the seven mission parameters also shown graphically in Fig. 2 and provides estimates for their uncertainties, the ranges in which their values are varied to produce Fig. 5, and the values assigned to them during our parameter estimation analyses. These parameters can be determined from measurements of the spacecraft position which are based on the spacecraft navigation system without involving the LPF optical readout [23,24]. The uncertainties on the navigation parameter values before the flight, i.e. before the trajectory for the transition from L1 to SP is chosen, and those determined during the flight are provided in columns three and four, respectively. The errors on the angle $\alpha$ that defines the orientation of the solar panel are below $1^{\circ}$ : as explained in Sec. III, we set $\alpha=90^{\circ}$ and the error may be neglected within the scope of this paper. Additionally, the time of closest approach to the SP is not included in the parameter list as it is of the order of several seconds and can be neglected with respect to the signal length. The values reported in the last column are those used for the analysis of the theory parameters. These numbers are based on [25] and [23]. While, recent investigations show that it may be possible to realize a trajectory directly through the SP, we have conservatively set $\|\xi\|=20 \mathrm{~km}$.

\begin{tabular}{lccccc}
\hline \hline Parameter & Description & Uncertainty before flight & Uncertainty after flight & Range & Value \\
\hline$\|\boldsymbol{\xi}\|$ & Fly-by distance & $5 \mathrm{~km}$ & $5 \mathrm{~km}$ & {$[0 ; 300] \mathrm{km}$} & $20 \mathrm{~km}$ \\
$\varphi$ & Trajectory polar angle & $30^{\circ}$ & $\ll 1^{\circ}$ & {$[0 ; 360]^{\circ}$} & $30^{\circ}$ \\
$\eta$ & Trajectory azimuthal angle & $30^{\circ}$ & $\ll 1^{\circ}$ & {$[0 ; 180]^{\circ}$} & $70^{\circ}$ \\
$\eta_{\perp}$ & Polar angle of the position of closest approach & uniform & $\|\xi\|$ dependent & {$[0 ; 180]^{\circ}$} & $90^{\circ}$ \\
$\operatorname{sign}\left(\sin \varphi_{\perp}\right)$ & Hemisphere of the position of closest approach & $\{-1,1\}$ & $\ldots$ & $\{-1,1\}$ & +1 \\
$\|\boldsymbol{v}\|$ & Spacecraft velocity & $0.1 \mathrm{~km} / \mathrm{s}$ & $1 \mathrm{~cm} / \mathrm{s}$ & {$[1 ; 2] \mathrm{km} / \mathrm{s}$} & $1.5 \mathrm{~km} / \mathrm{s}$ \\
$\beta$ & Orientation of the LPF sensitive axis & $30^{\prime}$ & $30^{\prime}$ & {$[0 ; 360]^{\circ}$} & $0^{\circ}$ \\
\hline \hline
\end{tabular}


In the case of optimal filtering, one searches for the filter that best fits the data. This provides a way to estimate the true signal template. In our study, fixing the true signal template a priori and building a filter upon it allows us to determine the dependency of the magnitude of the matched filter response to a signal with its parameters offset by $\Delta \lambda^{m}$. This is the measure generally used to quantify the resolution with which we can distinguish one template from another. With this in mind, we rewrite the filter in discrete form,

$$
c\left(\Delta \lambda^{m}\right)=c\left(\lambda_{0}^{m}, \lambda_{\mathrm{v}}^{m}\right)=\sum_{j=1}^{N} \frac{\tilde{h}\left(f_{j}, \lambda_{\mathrm{v}}^{m}\right) \tilde{h}^{*}\left(f_{j}, \lambda_{0}^{m}\right)}{S\left(f_{j}\right)} \Delta f_{j},
$$

where frequency indices cover the instrument frequency range and $\Delta f_{j}=f_{j+1}-f_{j}$, and we consider the ambiguity function built upon the linear filter as follows:

$$
\hat{c}\left(\lambda_{0}^{m}, \lambda_{\mathrm{v}}^{m}\right)=\frac{c\left(\lambda_{0}^{m}, \lambda_{\mathrm{v}}^{m}\right)}{\sqrt{c\left(\lambda_{0}^{m}, \lambda_{0}^{m}\right) c\left(\lambda_{\mathrm{v}}^{m}, \lambda_{\mathrm{v}}^{m}\right)}} .
$$

The ambiguity function is normalized to yield unity when the template matches the input signal and less than unity otherwise.

\section{SNR as a function of mission parameters}

Estimating the SNR as a function of the mission parameters provides insight into the optimal values these should take and allows us to identify any peculiar behavior of the templates over the parameter space. In turn, if no peculiarities emerge, we assume that this allows us to investigate the behavior of the signal in the neighborhood of a single, representative point of our choice in the parameter space and to extrapolate results over the whole range of parameter values. At this location of our choice, we investigate the behavior of the ambiguity function, as this allows us to assess how much reduction in SNR would be caused by deviations from the nominal mission parameter values.

We now compute the expected SNR for the two noise models - current best noise estimate and requirements noise-discussed in Sec. II C. The SNR values are calculated using Eq. (31) for 1000 different trajectories each with random parameter values uniformly sampled within the ranges given in the fifth column of Table I. As shown in Fig. 5, the Gaussian fits to the histograms of the SNR values peak at $\rho \simeq 23$ and $\rho \simeq 5$ for the current best noise estimate and the requirements estimate, respectively.

When varying the mission parameters sequentially within the predefined ranges, the remaining parameters are fixed to the values given in the last column of Table I.

The first parameter we vary is the sensitive axis orientation angle $\beta$. As seen in Fig. 6, the SNR is not very

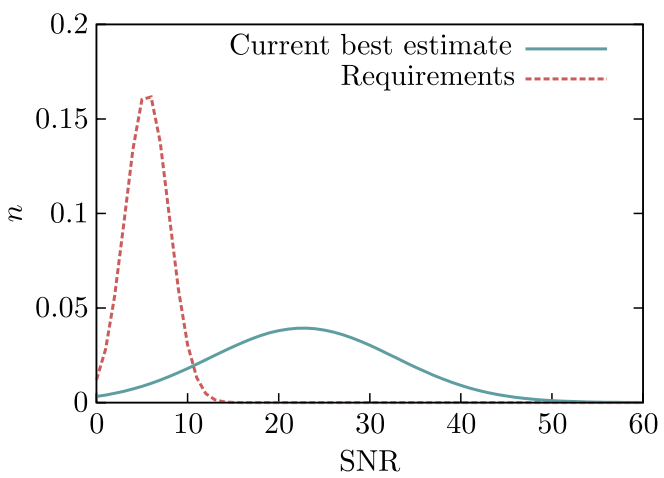

FIG. 5 (color online). Fraction of trajectories $n$ with SNR value specified on the horizontal axis. The SNR was calculated using 1000 trajectories with randomly varied parameters for both the current best noise estimate and the requirements noise. The parameter values were uniformly sampled over the ranges given in the fifth column of Table I. The curves are the Gaussian fits to the discrete distributions that were obtained.

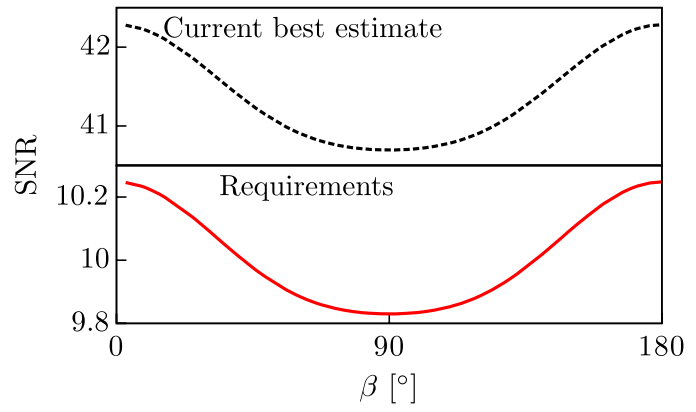

FIG. 6 (color online). SNR as a function of the orientation angle of the sensitive axis $\beta$ for the two noise realizations. The remaining mission parameters are fixed according to the set of values given in the last column of Table I.

sensitive to the choice of $\beta$ and that the optimal value for $\beta$ for both noise realizations is $\beta=0^{\circ}$ or $\beta=180^{\circ}$. We will thus fix $\beta=0^{\circ}$ for the analysis and for the experiment planning.

The SNR exhibits a smooth behavior also when the flyby distance and the spacecraft velocity are varied, as shown in Figs. 7 and 8, respectively. We notice that, as is to be expected, the closer LPF flies to the SP, the higher the SNR is, because tidal stress deviations are stronger, whereas the specific value of the spacecraft velocity is not very crucial in the interval reported in Table I.

Similarly, the SNR is smooth in the $\varphi-\eta$ subspace, as shown in Fig. 9. These are the two angles that define the orientation of the spacecraft trajectory. While the SNR is flat in $\varphi$, it is maximum for $\eta=\left\{90^{\circ}, 270^{\circ}\right\}$. In these specific cases we see that more SNR is accumulated if LPF flies within the ecliptic plane and that the direction of flight within this plane has minimal influence.

As the range of values covered by $\eta_{\perp}$ depends on the combination of other parameter values via Eq. (12), $\eta_{\perp}$ 


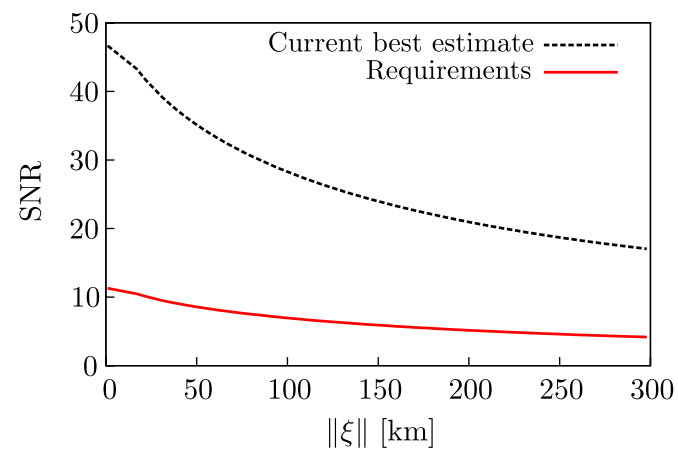

FIG. 7 (color online). SNR as a function of the distance from the SP $\|\xi\|$ for the two noise realizations. The remaining parameters are fixed according to the set of values given in the last column of Table I.

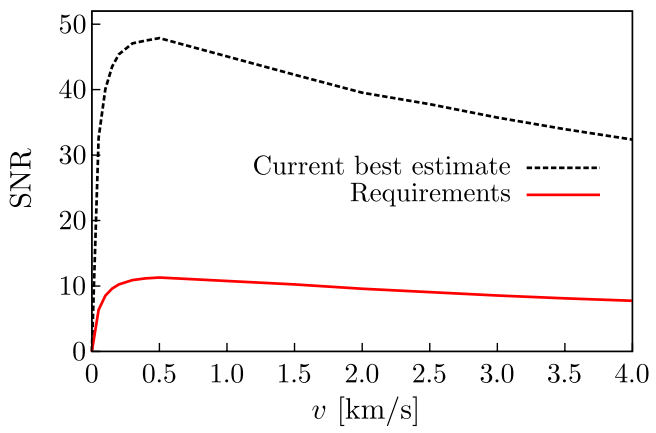

FIG. 8 (color online). SNR as a function of the spacecraft velocity $v$ for the two noise realizations. The remaining parameters are fixed according to the set of values given in the last column of Table I. We vary the values of velocity within the larger range than given in the Table I, i.e. from 0 to $4 \mathrm{~km} / \mathrm{s}$, to observe the maximum of SNR.

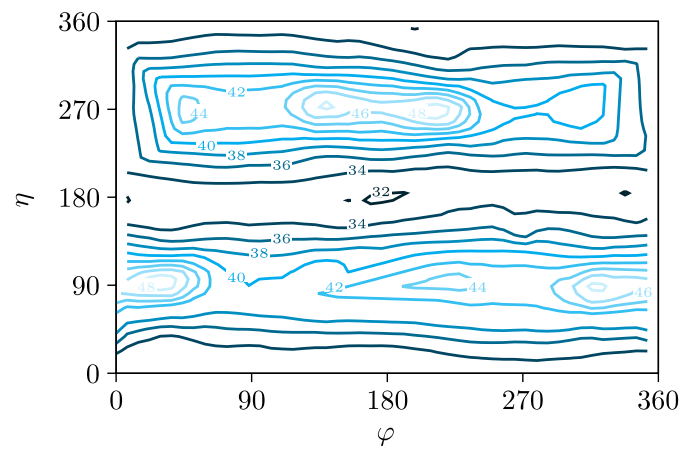

FIG. 9 (color online). SNR as a function of the angles $\varphi$ and $\eta$ that determine the direction of the trajectory. The SNR estimates are plotted for the current best noise estimate. The behavior for the requirements noise is similar, but with magnitudes in the range $[8 ; 12]$. The remaining parameters are fixed to the set of values given in the last column of Table I. cannot span the whole interval $[0,180]^{\circ}$ for a specific choice of $\eta$ and $\varphi$. Therefore, we do not present SNR estimates as a function of $\eta_{\perp}$. We note, however, that in the cases we considered the dependence of the SNR on $\eta_{\perp}$ is weak.

\section{SNR loss due to mismatched mission parameters}

Having established the dependence of the SNR on the mission parameter space, we may now study the loss of SNR as a function of parameter mismatch within the known navigation uncertainties on the mission parameters. As discussed previously, we fix $\beta=0^{\circ}$. At the same time, even though $\eta$ has its highest SNR estimate for $\eta=90^{\circ}$, we will choose it to be $\eta=70^{\circ}$ in order to avoid performing our analyses in the best case scenario. Contrary to the alignment of the LPF sensitive axis, the value of $\eta$ depends on the maneuvers that are necessary for LPF to leave the Lissajous orbit around the first Lagrangian point. Further, the option of multiple fly-by's [23] implies different estimates for the angle values. We therefore keep this parameter away from its optimal value during our analyses and avoid choosing a trajectory within the ecliptic plane.

Hereafter, we proceed by taking one-dimensional slices through the parameter space, fixing six parameters out of seven to the values listed in the last column of Table I. The parameters are varied only around their true values, i.e. the values listed in Table I, which we treat as the parameters of the signal buried in the data. All parameters are varied within intervals that include the spacecraft navigation errors listed in Table I. Similarly to what we did for SNRs, we estimate the ambiguity function [Eq. (33)] between templates with varied parameter values and the template with all parameters set to the values listed in Table I. When the ambiguity function varies very little, we can assume the parameters are essentially exactly known and can be fixed during the analysis of the theory parameters.

Our results for the fly-by distance $\|\boldsymbol{\xi}\|$ are shown in Fig. 10. The true values of the mission parameters follow

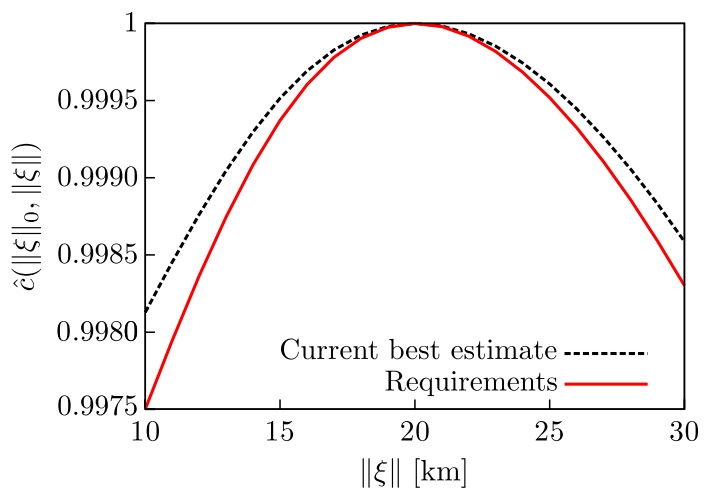

FIG. 10 (color online). Ambiguity function for the fly-by distance $\|\xi\|$ for the two noise realizations. The true value of the parameter is $\|\xi\|_{0}=20 \mathrm{~km}$. The remaining parameters are fixed according to the set of values given in the last column of Table I. 
Table I, so that $\|\boldsymbol{\xi}\|_{0}=20 \mathrm{~km}$. Templates were evaluated between $\|\boldsymbol{\xi}\|=10 \mathrm{~km}$ and $\|\boldsymbol{\xi}\|=30 \mathrm{~km}$ every $1 \mathrm{~km}$ and the ambiguity function $\hat{c}\left(\|\boldsymbol{\xi}\|_{0},\|\boldsymbol{\xi}\|\right)$ was calculated correspondingly, using both LPF noise curves. We find that if the fly-by distance is mismatched by less then $5 \mathrm{~km}$, i.e. the navigation error before the flight reported in Table I, the ambiguity function is greater than 0.999 . We conclude that we can fix this parameter to $20 \mathrm{~km}$ for future analyses and that it does not need to be estimated from the LPF measurement, but can instead be determined via the spacecraft navigation system.

The same conclusion holds for the spacecraft velocity $v$. We set $v_{0}=1.5 \mathrm{~km} / \mathrm{s}$ to be the true value of the parameter and calculate the ambiguity function $\hat{c}\left(v_{0}, v\right)$ varying $v$ between $1.0 \mathrm{~km} / \mathrm{s}$ and $2 \mathrm{~km} / \mathrm{s}$ and sampling it every $0.1 \mathrm{~km} / \mathrm{s}$. The results are shown in Fig. 11 for both LPF noise realizations. As is evident, templates are more sensitive to velocity uncertainties and variations. However, $\hat{c}\left(v_{0}, v\right)>0.998$ for velocity variations within $0.1 \mathrm{~km} / \mathrm{s}$, which is the value reported in Table I for the uncertainty before the flight. Further, $v$ may be determined during the flight with an uncertainty of $1 \mathrm{~cm} / \mathrm{s}$, so we assume this parameter to be fixed at $1.5 \mathrm{~km} / \mathrm{s}$ during future analyses.

Next, we vary the angles $\varphi$ and $\eta$ that determine the orientation of the spacecraft trajectory. Our results for the ambiguity function are presented in Fig. 12. The true parameter values are $\varphi_{0}=30^{\circ}$ and $\eta_{0}=70^{\circ}$. We consider an interval of $10^{\circ}$ around both values and sample each interval every $1^{\circ}$. The contours shown in the figure are for the current best noise estimate. The elongation relative to the ecliptic changes the template more than the angle that defines the inclination to the line connecting the Earth and the Sun. Despite the big uncertainty in these parameters before the experiment (see Table I), the errors on the determination of these parameters during flight are very small $\left(\ll 1^{\circ}\right)$, so that they, too, may be assumed to be fixed to their true values for future analyses. The result for the requirements noise is very similar to the result for the

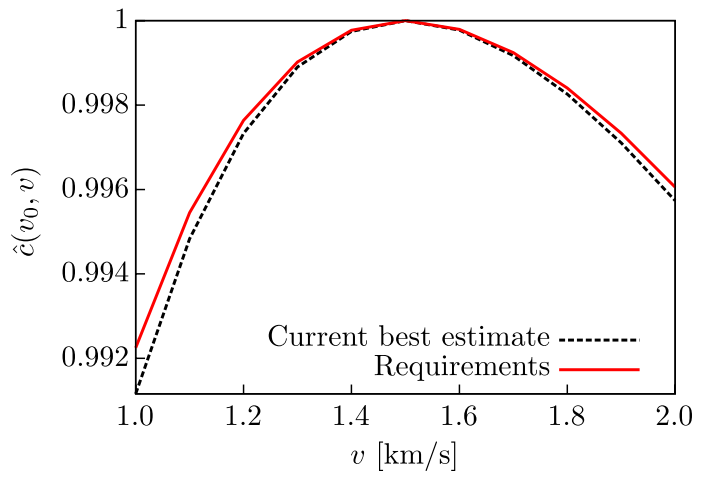

FIG. 11 (color online). Ambiguity function for the spacecraft velocity $v$ for the two noise realizations. The true value of the parameter is $v_{0}=1.5 \mathrm{~km} / \mathrm{s}$. The remaining parameters are fixed according to the set of values given in the last column of Table I.

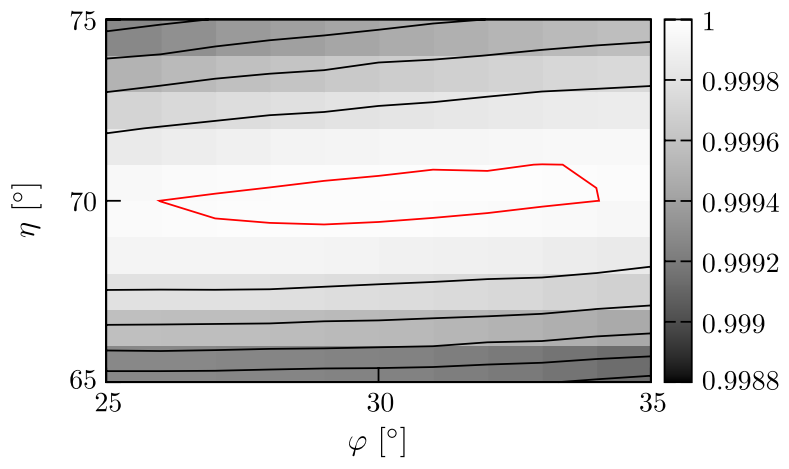

FIG. 12 (color online). Two-dimensional ambiguity function for the angles $\varphi$ and $\eta$ that determine the direction of the spacecraft trajectory. The results are obtained with the current best noise estimate model. The true values of the parameters are set to $\varphi_{0}=30^{\circ}$ and $\eta_{0}=70^{\circ}$ and the remaining parameters are fixed according to the set of values given in the last column of Table I. Both angles are varied with steps of $1^{\circ}$. The closed contour indicates the location of $\hat{c}=0.99998$.

current best noise estimate, therefore we will not display them here.

Finally we consider the position of the perpendicular to the trajectory determined by $\operatorname{sign}\left(\sin \varphi_{\perp}\right)$ and $\eta_{\perp}$. For $\operatorname{sign}\left(\sin \varphi_{\perp}\right)$ there will be no uncertainty after the flight and for the $\eta_{\perp}$ the results are presented in Fig. 13 . They show that the signal templates are not sensitive to variations of this angle.

To summarize, we picked a specific location in the mission and theory parameter space and investigated the behavior of the ambiguity function. Within the predicted uncertainties on the mission parameters reported in Table I, the ambiguity function drops minimally compared to the case of exactly matching templates. By assuming that this is the case for all other possible parameters space locations, we make the accurate approximation that the mission

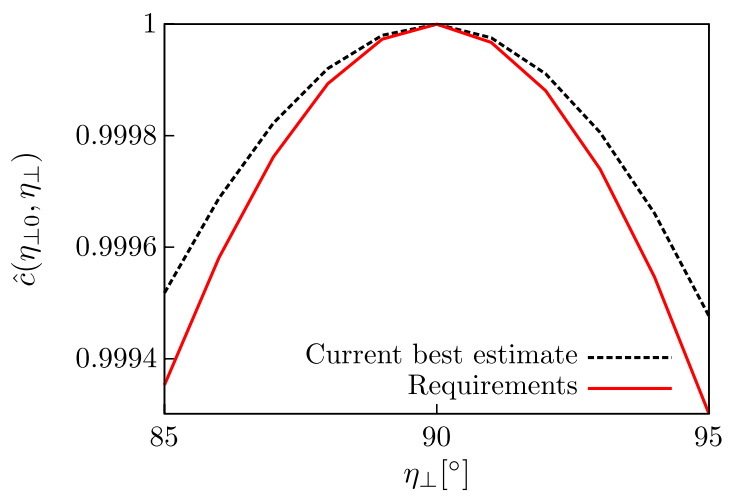

FIG. 13 (color online). Ambiguity function for the angle $\eta_{\perp}$ which defines the position of the perpendicular to the trajectory for the two noise realizations. The true value of the parameter is $\eta_{\perp}=90^{\circ}$ The remaining parameters are fixed according to the set of values given in the last column of Table I. 
parameters can be assumed to be "known" without any loss of generality. They are no longer search parameters, which leaves only the theory parameters as unknowns and as the sole target of the search. The analysis of the theory parameters will therefore not require the mission parameters to be measured, nor will it need them to be considered during parameter estimation and model selection. In other words, we can factor the mission parameters out of the theory parameter analyses.

Additionally, we were able to determine the optimal values of $\beta$ - the LPF sensitive axis orientation-and $\eta$ the angle between the spacecraft trajectory and the perpendicular to the ecliptic plane. In the latter case, we showed that the optimal trajectory lies in the plane of the ecliptic.

\section{Analysis of the theory parameters}

We now discuss the data analysis framework to study the signal predicted by various alternative theories of gravity. We apply this framework to the case of the $\mathrm{TeV} e \mathrm{~S}$ theory. More specifically, having fixed an interpolating function $\mu$, we study the $\left(k, a_{0}\right)$ parameter space, where $k$ is a dimensionless coupling parameter and $a_{0}$ is a characteristic acceleration scale (see Sec. IV B 2). We introduce a parameter estimation method based on a Bayesian approach. With this method, information regarding the parameters of the theory can be extracted from the data. Further, we exploit Bayes' theorem to perform model selection, choosing between the hypothesis of having a signal in the noise and the null hypothesis according to which the data consists of noise only.

We discuss how parameter estimation results can be assessed in the case of absence of a signal and how this allows us to rule out portions of the parameter space. Finally, we show how model selection can be applied to realistic data that contains noise artifacts. The results of this study will show whether a glitch in the data can be misinterpreted as a signal and where this will be localized in the parameter space.

\section{Bayesian parameter estimation}

Following Bayes' theorem, the posterior distribution $p\left(k, a_{0} \mid\{\tilde{x}\}, I\right)$ of $k$ and $a_{0}$ given the data $\{\tilde{x}\}$ and the relevant background information $I$ reads

$$
p\left(k, a_{0} \mid\{\tilde{x}\}, I\right)=\frac{p\left(\{\tilde{x}\} \mid k, a_{0}, I\right) p\left(k, a_{0} \mid I\right)}{p(\{\tilde{x}\} \mid I)},
$$

where $p\left(k, a_{0} \mid I\right)$ is the prior distribution on the parameters, $p\left(\{\tilde{x}\} \mid k, a_{0}, I\right)$ is the likelihood, and $p(\{\tilde{x}\} \mid I)$ is the Bayesian evidence, which is the marginal probability density of the data and normalizes the posterior. The data model is the sum of a deterministic signal and Gaussian noise and is computed in the frequency domain, as described in Sec. IV A. We therefore write the likelihood of the Fourier transformed data $\{\tilde{x}\}$ as $p\left(\{\tilde{x}\} \mid k, a_{0}, I\right)=\prod_{j=1}^{N / 2} \frac{1}{\sigma_{j}^{2} 2 \pi} \exp \left(-\frac{\left|\tilde{x}_{j}-\tilde{h}_{j}\left(k, a_{0}\right)\right|^{2}}{2 \sigma_{j}^{2}}\right)$,

where $N$ is the number of samples over the measurement time interval. In this expression, the variance of the noise $\sigma_{j}^{2}$ is calculated from the (PSD) normalized by the width of the frequency bin $\sigma_{j}^{2}=S\left(f_{j}\right) / \Delta f$ [see Eq. (16)]. The noise model is based on the theoretical estimates of the noise for LPF (see Fig. 1). In writing the expression for the likelihood, we assumed that each frequency bin is statistically independent, so that the likelihood can be written as the product of bivariate Gaussian probability density functions.

As a result of the parameter estimation, we shall obtain a joint posterior distribution for parameters $k$ and $a_{0}$. However, we are also interested in estimating each parameter separately after performing the experiment. To obtain the posterior distribution of each parameter separately, we marginalize the joint distribution for the two parameters over the other parameter, i.e.

$$
\begin{aligned}
& p(k \mid\{\tilde{x}\}, I)=\int_{-\infty}^{\infty} p\left(k, a_{0} \mid\{\tilde{x}\}, I\right) \mathrm{d} a_{0}, \\
& p\left(a_{0} \mid\{\tilde{x}\}, I\right)=\int_{-\infty}^{\infty} p\left(k, a_{0} \mid\{\tilde{x}\}, I\right) \mathrm{d} k .
\end{aligned}
$$

These marginal distributions represent our belief in a specific value of one of the two parameters and yield the uncertainty on the parameter estimate following the experiment.

\section{Prior space}

As a first step to set priors in the $\left(k, a_{0}\right)$ parameter space, we restrict it using the following considerations. We assume that, within some precision, the gradient of the gravitational potential is Newtonian in the nonrelativistic limit at a distance from the SP equal to the distance from the SP to the Earth. The gradient of the non-Newtonian potential at this distance depends on the parameters $k$ and $a_{0}$ and allows us, therefore, to impose restrictions on the combination of these parameters. Eq. (19), which governs the non-Newtonian potential $\phi$, depends on the $\mu$ function, which goes to unity in the Newtonian limit, when its argument becomes sufficiently large. Taking the definition ${ }^{2}$ of the interpolating function $\mu$ given in Eq. (23) and expanding it in the $|\nabla \Phi| / a_{0} \gg 1$ limit, when $\mu \rightarrow 1$, we obtain

$$
y=\frac{3}{4(1-\mu)}+\mathcal{O}(1-\mu)
$$

\footnotetext{
${ }^{2}$ We remark that the interpolating function used in the numerical calculations defined in Eq. (24) and the one expanded here correspond in the limit we consider, as shown in Fig. 3.
} 
Equations (17) and (26) can then be used to express the argument of the $\mu$ function as

$$
y \equiv k l^{2}|\nabla \phi|^{2}=\frac{k^{3} l^{2}}{16 \pi^{2}}|\nabla \Phi|^{2}
$$

where higher order corrections in $(k / 4 \pi)$ are neglected. Combining the last two results and expressing $l$ in terms of $a_{0}$ as in Eq. (20) yield

$$
\mu \approx 1-\frac{64 \pi^{4}}{k^{4}} \frac{a_{0}^{2}}{|\nabla \Phi|^{2}}+\mathcal{O}\left(y^{-2}\right) .
$$

If we fix an admissible error $\varepsilon^{2}$ on deviations of $\mu$ from unity, we readily obtain the constraint

$$
\frac{a_{0}}{|\nabla \Phi|}<\frac{k^{2}}{8 \pi^{2}} \varepsilon
$$

Imposing this restriction allows one to exclude certain combinations of $k$ and $a_{0}$.

In our analysis, we set $\varepsilon=10^{-5}$, and the resulting, restricted parameter space is shown in Fig. 14. This is a conservative value compared to the latest boundaries imposed on the precision of the additional acceleration allowed in the Solar System [28]. We do not take into account such stringent requirements, as we want to develop and illustrate a data analysis scheme that does not automatically depend on other astronomical restrictions of the parameter space.

We consider a uniform prior parameter distribution (known as flat or constant prior) for the theory parameters. We thus set the prior for $a_{0}$ and $k$ to be flat in the admissible portion of the parameter space $\mathcal{P}$, the area of which is given by

$$
\mathcal{A}=\int_{0}^{k^{\mathrm{min}}} a_{0}^{\prime}(k) \mathrm{d} k+\left(k^{\mathrm{max}}-k^{\min }\right)\left(a_{0}^{\max }-a_{0}^{\min }\right),
$$

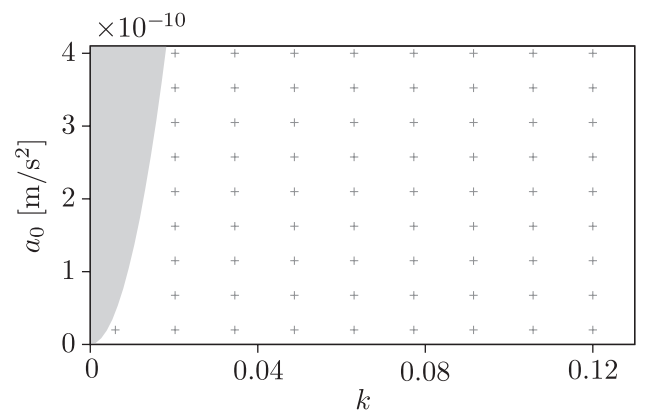

FIG. 14. The $\left(k, a_{0}\right)$ parameter space. The shaded area represents the part of the parameter space ruled out by Eq. (40). Crosses indicate points where Eq. (19) was solved numerically. Signal templates are built upon these solutions and are used, in turn, to determine signal templates at a generic point $\left(k, a_{0}\right)$ via bicubic interpolation. where $k^{\min }$ is the value for which $a_{0}^{\prime}\left(k^{\min }\right)=a_{0}^{\max }=4 \times$ $10^{-10} \mathrm{~m} / \mathrm{s}^{2}$ and $a_{0}^{\prime}(k)$ is a solution of Eq. (40). Moreover, the values of the modified stress tensor are set at the lower boundary of the parameter space $a_{0}^{\min }=0$ to $\partial^{2} \phi /$ $\partial x^{i} \partial x^{j}=0$. It reflects the (GR) limit of $\mathrm{TeV} e \mathrm{~S}$ that can be obtained when $l \rightarrow \infty$ [20]. Equation (20) shows that this corresponds to $a_{0} \rightarrow 0$. We therefore have

$$
p\left(k, a_{0} \mid I\right)= \begin{cases}1 / \mathcal{A} & \left(k, a_{0}\right) \in \mathcal{P} \\ 0, & \text { otherwise }\end{cases}
$$

Flat priors depend on no underlying knowledge on the parameters, except the assumptions made on their span. As discussed in Sec. IV B 2, the ranges for the theory parameters are chosen here on the basis of astrophysical observations [21] and in order to keep the theory consistent [20].

As we consider a constant prior, with the exception of the prior boundary constraints, the shape of the posterior parameter distributions will be dictated only by the likelihood function. We note that our Bayesian analysis scheme allows for more physically realistic priors which opens a way for the future analyses of different theoretical models.

\section{E. Model selection}

The framework for model selection that we develop here is based on the Bayesian approach to model selection and can be applied to a variety of hypotheses. For example, we can test a model that assumes the data is the sum of a signal and Gaussian noise, a model that assumes that the data is Gaussian noise only, a model that assumes the data is nonGaussian noise, a model that assumes Gaussian noise with glitches, and so forth.

Any number of models $\mathcal{M}_{i}$ can be defined and Bayes' theorem [see Eq. (34)] can be directly applied as follows:

$$
p\left(\mathcal{M}_{i} \mid\{\tilde{x}\}, I\right)=\frac{p\left(\{\tilde{x}\} \mid \mathcal{M}_{i}, I\right) p\left(\mathcal{M}_{i} \mid I\right)}{p(\{\tilde{x}\} \mid I)} .
$$

This expression tells us how to determine the posterior probability $p\left(\mathcal{M}_{i} \mid\{\tilde{x}\}, I\right)$, which is the probability of the $i$ th model $\mathcal{M}_{i}$ being correct, given the data $\{\tilde{x}\}$ and the background information $I$. The denominator is the Bayesian evidence, a normalization term that reads

$$
p(\{\tilde{x}\} \mid I)=\sum_{i} p\left(\{\tilde{x}\} \mid \mathcal{M}_{i}, I\right) p\left(\mathcal{M}_{i} \mid I\right),
$$

where $p\left(\{\tilde{x}\} \mid \mathcal{M}_{i}, I\right)$ is the evidence for the model $\mathcal{M}_{i}$ and $p\left(\mathcal{M}_{i} \mid I\right)$ is the model prior.

To properly normalize the model posterior distribution, however, one must know all possible models in order to compute Eq. (44) and hence Eq. (43). This may be avoided by considering the ratio between model posteriors, usually referred to as posterior odds ratio. For two models $\mathcal{M}_{1}$ and $\mathcal{M}_{2}$, this reads 


$$
\frac{p\left(\mathcal{M}_{1} \mid\{\tilde{x}\}, I\right)}{p\left(\mathcal{M}_{2} \mid\{\tilde{x}\}, I\right)}=\frac{p\left(\{\tilde{x}\} \mid \mathcal{M}_{1}, I\right)}{p\left(\{\tilde{x}\} \mid \mathcal{M}_{2}, I\right)} \frac{p\left(\mathcal{M}_{1} \mid I\right)}{p\left(\mathcal{M}_{2} \mid I\right)} .
$$

The ratio between the evidences for the two models appearing on the right-hand side of the equation is called the Bayes factor. The second fraction on the same side of the equation, $p\left(\mathcal{M}_{1} \mid I\right) / p\left(\mathcal{M}_{2} \mid I\right)$, is the prior model odds. The posterior odds ratio represents our confidence in one model against the other, based on the data and the background information $I$. Here $p(\{\tilde{x}\} \mid \mathcal{M}, I)$ is the likelihood marginalized over its entire parameter space for each model.

As our goal is to quantify our confidence in signal detection, we introduce two ways to model the measured data. The first model, labeled $\mathcal{S}$, describes the data as the sum of a signal and of Gaussian noise, i.e.

$$
\tilde{x}_{j}=\tilde{h}_{j}+\tilde{n}_{j}
$$

The second model, with label $\mathcal{N}$, describes the data as Gaussian noise only, that is,

$$
\tilde{x}_{j}=\tilde{n}_{j}
$$

The ratio between the $\mathcal{S}$ and $\mathcal{N}$ model posteriors is thus

$$
\frac{p(\mathcal{S} \mid\{\tilde{x}\}, I)}{p(\mathcal{N} \mid\{\tilde{x}\}, I)}=\frac{p(\{\tilde{x}\} \mid \mathcal{S}, I)}{p(\{\tilde{x}\} \mid \mathcal{N}, I)} \frac{p(\mathcal{S} \mid I)}{p(\mathcal{N} \mid I)} .
$$

The Bayesian evidence for a model is calculated by integrating the joint probability density for the data and parameters over the parameter space of the model. In our MOND example, the evidence for the $\mathcal{S}$ model reads

$$
\begin{aligned}
p(\{\tilde{x}\} \mid \mathcal{S}, I) & =\iint_{\mathcal{P}} p\left(\{\tilde{x}\}, k, a_{0} \mid \mathcal{S}, I\right) d k d a_{0} \\
& =\iint_{\mathcal{P}} p\left(\{\tilde{x}\} \mid k, a_{0}, \mathcal{S}, I\right) p\left(k, a_{0} \mid \mathcal{S}, I\right) \mathrm{d} k \mathrm{~d} a_{0} .
\end{aligned}
$$

This is a weighted integral of the likelihood, $p\left(\{\tilde{x}\} \mid \lambda_{0}^{t}, \mathcal{S}, I\right)$ [see Eq. (49)], over the space of unknown parameters, where the weights are set by the prior distributions of the theory parameters, $k$ and $a_{0}$ in this case. The Bayesian evidence thus depends on the volume of the parameter space and on the priors. If the dimensionality of the parameter space is large, or if the likelihood and/or the prior are strongly localized, calculating this integral on a uniform grid in the parameter space can become computationally costly. A more practical solution to the problem is to randomly sample the parameter space. To compute the integral in Eq. (49), we use the Nested Sampling algorithm, which was specifically designed to calculate evidence values [29].
For the $\mathcal{N}$ model, there are no theory parameters to marginalize over, i.e. the theory parameter space is dimensionless $\left(\lambda_{0}^{t}=\{\varnothing\}\right)$. The evidence is thus simply the noise likelihood,

$$
p(\{\tilde{x}\} \mid \mathcal{N}, I)=\prod_{j=1}^{N / 2} \frac{1}{\sigma_{j}^{2} 2 \pi} \exp \left[-\frac{\left|\tilde{x}_{j}\right|^{2}}{2 \sigma_{j}^{2}}\right] .
$$

The difference between the likelihoods for models $\mathcal{S}$ and $\mathcal{N}$, Eqs. (49) and (50), respectively, is that in the latter the Gaussian noise is expressed as $\tilde{n}_{j}=\tilde{x}_{j}$, while in the former $\tilde{n}_{j}=\tilde{x}_{j}-\tilde{h}_{j}$. The likelihood for model $\mathcal{N}$ can thus be viewed as the likelihood for model $\mathcal{S}$ with the signal amplitude set to zero. For the Bayes factor in Eq. (48), the likelihood normalization terms cancel out, which simplifies the calculations, leaving only the exponentials of the likelihoods and the normalization due to the model priors. The ratio of the model priors represents our confidence in one model against the other, based on the background information $I$. In the absence of preference for either model, this ratio is set to unity, while if background information is available, it can be included in the prior odds ratio accordingly. We will not prioritize a model over the other, so that the posterior odds ratio is simply equal to the Bayes factor.

The posterior odds ratio discussed in this section can be used to decide whether there was a signal buried in the data gathered during the SP fly-by and to provide a quantitative measure of our confidence in a signal detection.

\section{RESULTS}

We test our data analysis method on artificially simulated data to assess the performance of the framework and inspect the various possible outcomes of the experiment. In order to justify the experiment feasibility, it is important to establish what conclusions can be made on the basis of data acquired during the LPF flight. More specifically, we check the implementation of the parameter estimation and model selection, and determine how well the parameter values may be inferred and what choices about the model that best describes the data may be made.

The artificial data is generated following the model defined in Eq. (15) and consists of the signal with additive Gaussian noise characterized by the known ASD of the instrument noise (see Fig. 1). The real and imaginary parts of the noise $\tilde{n}(f)$ are treated as statistically independent and drawn from a Gaussian distribution with the given $\sigma^{2}(f)$ providing

$$
\begin{aligned}
p(\tilde{n}(f)) & =p(\Re[\tilde{n}(f)]) p(\Im[\tilde{n}(f)]) \\
& =\frac{1}{2 \pi \sigma^{2}(f)} \exp \left(-\frac{\Re[\tilde{n}(f)]^{2}+\Im[\tilde{n}(f)]^{2}}{2 \sigma^{2}(f)}\right) .
\end{aligned}
$$

For the signal model $\tilde{h}\left(\lambda_{0}^{m}, \lambda_{0}^{t}\right)$ we chose a particular theoretical prediction for the deviations of the gravity stress 
tensor from the Newtonian case, as discussed in Sec. IV B. We test our data analysis setup on $\mathrm{TeV} e \mathrm{~S}$, but we wish to emphasize that this analysis framework is general and can be used for any signal predictions.

As shown in Sec. IV C, the mission parameters can be fixed and do not cause the signal to vary significantly once they are defined and measured. Throughout the analysis of the theory parameters, we fix a specific set of mission parameters values in accordance with Table I. We may thus write

$$
\tilde{h}\left(f_{j}, \lambda_{0}^{m}, \lambda_{0}^{t}\right)=\tilde{h}\left(f_{j}, \lambda_{0}^{m}, k, a_{0}\right)=\tilde{h}\left(f_{j}, k, a_{0}\right) .
$$

The theory parameter space $\left(k, a_{0}\right)$ was discussed in Sec. IV B 2 and SNRs are calculated following Eq. (31). Figure 15 shows the SNRs for the chosen LPF trajectory as a function of $\left(k, a_{0}\right)$. For large values of both $k$ and $a_{0}$ the SNR reaches values of $\sim 100$ for the current best estimate and $\sim 20$ for the requirements noise. This implies that the posterior distributions for the parameter estimates will be reasonably narrow in those high SNR regions. Conversely, we expect signals residing in low SNR areas to have correspondingly broader posterior probabilities.

Given the SNR estimates shown in Fig. 15, we choose a number of representative points in the parameter space with high, intermediate, and low SNR values, and estimate their posterior probabilities. These points are listed in Table II. We start with point 1 , for which $k$ and $a_{0}$ take their "standard" values [14]. This point belongs to the high SNR region. To test the area with the loudest SNRs, we probe point 2 . A third interesting region, where the performance of our interpolation must be checked, is the area near the boundary that was imposed on the prior parameter space [Eq. (40)]. We chose two points here: point 3 and point 4 for low and high SNRs, respectively. Further, we consider

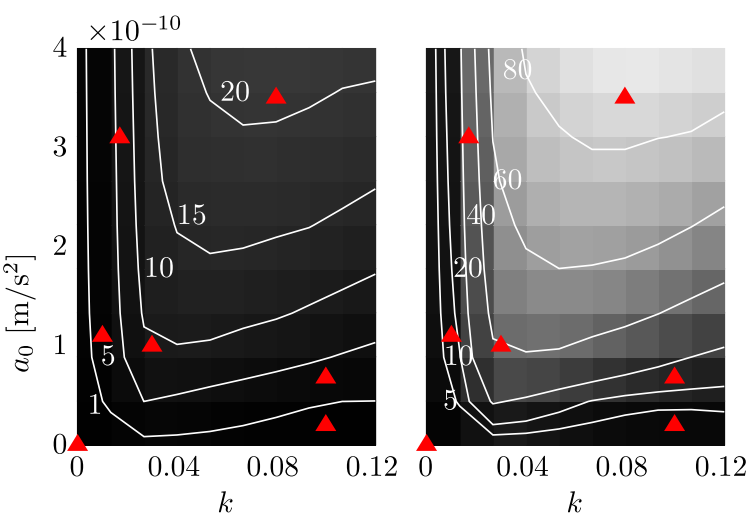

FIG. 15 (color online). SNR estimates for the current best noise estimate (right panel) and the requirements noise (left panel). The SNRs are calculated at the points in parameter space where the $\mathrm{T} e \mathrm{~V} e \mathrm{~S}$ numerical calculations were performed. The triangles correspond to the values of $k$ and $a_{0}$ for which the signal templates were injected into the data (see Table II).
TABLE II. Values of $k$ and $a_{0}$ for which the signal template was injected in the data to probe parameter estimation.

\begin{tabular}{llc}
\hline \hline Number & \multicolumn{1}{c}{$k$} & $a_{0}\left[10^{-10} \mathrm{~m} / \mathrm{s}^{2}\right]$ \\
\hline 1 & 0.030 & 1.00 \\
2 & 0.080 & 3.50 \\
3 & 0.010 & 1.10 \\
4 & 0.017 & 3.10 \\
5 & 0.100 & 0.20 \\
6 & 0.100 & 0.68 \\
7 & 0 & 0 \\
\hline \hline
\end{tabular}

two points with low SNRs: point 5 and point 6 . They are chosen relatively close to each other in order to assess the area where the transition from the detectable to nondetectable signal might occur. Finally, we consider point 7 , where the Newtonian limit of the theory lies and we expect to find no signal in the data. For each chosen point on the parameter space we perform 200 simulations with different noise realizations.

\section{A. Parameter estimation}

The experiment can give us insight into how well the parameters of the theory can be recovered and constrained from the data. This can be achieved by calculating the posterior probability distribution for the parameters. We have an initial prior assumption for the parameter values, which in our case is a simple uniform distribution over the predefined parameter space discussed in Sec. IV B 2. We compute evidence values using a random sampling algorithm (Nested Sampling [30,31]) as a mean to overcome potential issues due to the sampling of the theory parameter space, or to its high dimensionality. While the theory parameter space is two dimensional in our example, we must be ready to consider theories with a higher number of parameters. The algorithm and its specific implementation we used, MultiNest [32], are designed to efficiently sample a parameter space and to output the samples from the joint posterior parameter distribution and the Bayesian evidence.

To quantitatively summarize the information on the posterior distributions of the parameters, it is natural to use confidence intervals. These indicate the parameter range within which the area enclosed under the posterior has a certain probability. This provides an estimate on how confident we are that the value of a parameter falls in that range. As is customary, use the confidence interval values $68 \%, 95 \%$, and $99 \%$, which correspond to $1 \sigma, 2 \sigma$, and $3 \sigma$ deviations of a parameter from its mean value in the special case of a one-dimensional Gaussian distribution. Accordingly, we define the confidence contours

$$
\begin{aligned}
P\left(\left(k, a_{0}\right) \subset S, I\right) & =\iint_{S} p\left(k, a_{0} \mid\{x\}, I\right) \mathrm{d} k \mathrm{~d} a_{0} \\
& =(68 \%, 95 \%, 99 \%),
\end{aligned}
$$



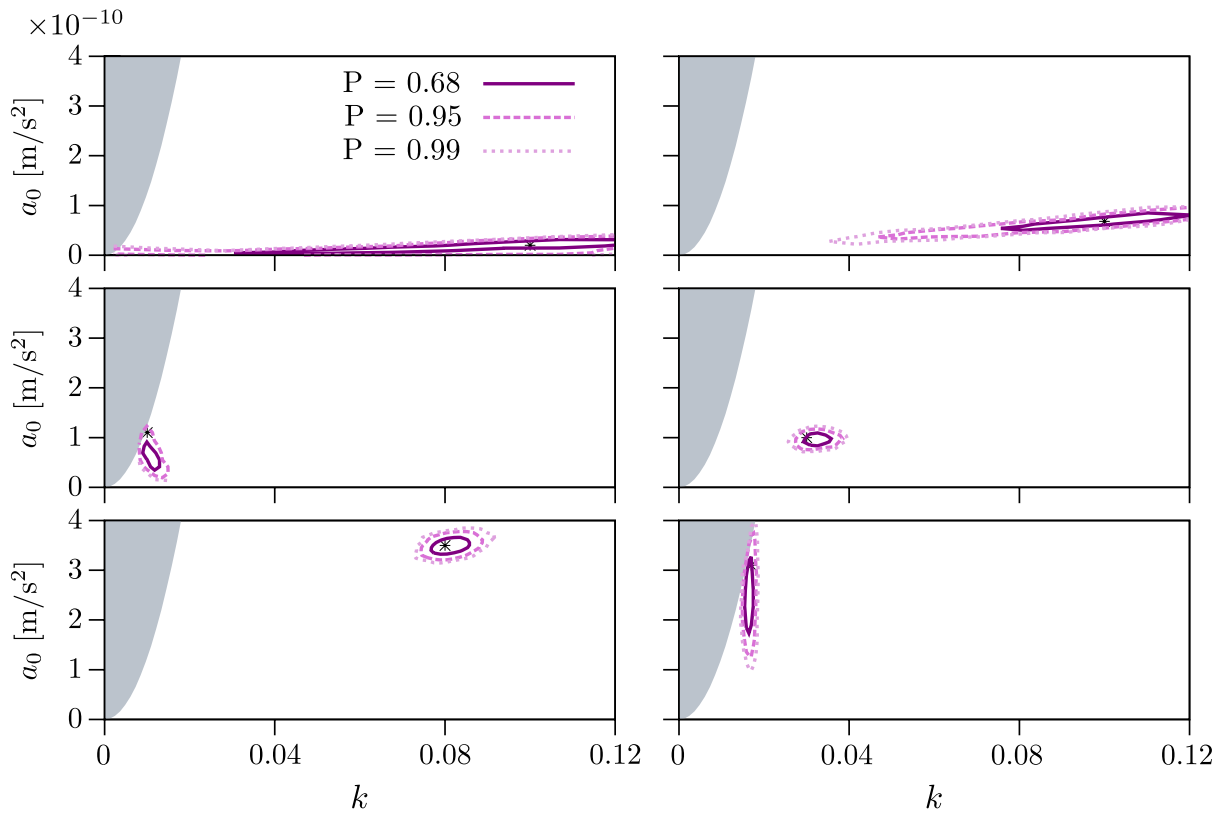

FIG. 16 (color online). Joint posterior probability distribution for the parameters $k$ and $a_{0}$ using the current best estimate noise model. Contours represent lines of constant probability density defining regions that enclose $68 \%, 95 \%$, and $99 \%$ of the probability. The panels represent six signal injections at the first six points in the parameter space listed in Table III.

where the space $S$ corresponds to the minimal volume underneath the posterior probability that integrates to predefined probability. The resulting contours also represent lines of constant probability density. Figure 16 shows the contour plots of the joint posterior distributions for the parameters $k$ and $a_{0}$ for simulated signals located at selected parameter space positions.

The resulting estimates of the posterior probabilities are shown in Figs. 16 and 17 for the current best estimate noise and for the requirements noise, respectively. The results are presented for a single noise realization. Estimates for the standard deviation of the posterior distributions of $k$ and $a_{0}$ averaged over 200 noise realizations for the current best noise estimate and requirements noise are given in Table III. For signals with high SNRs (see Fig. 15) the posterior likelihoods are narrow and exhibit low correlation between the two parameters. This means that in the case of signal detection it would be possible to estimate them with
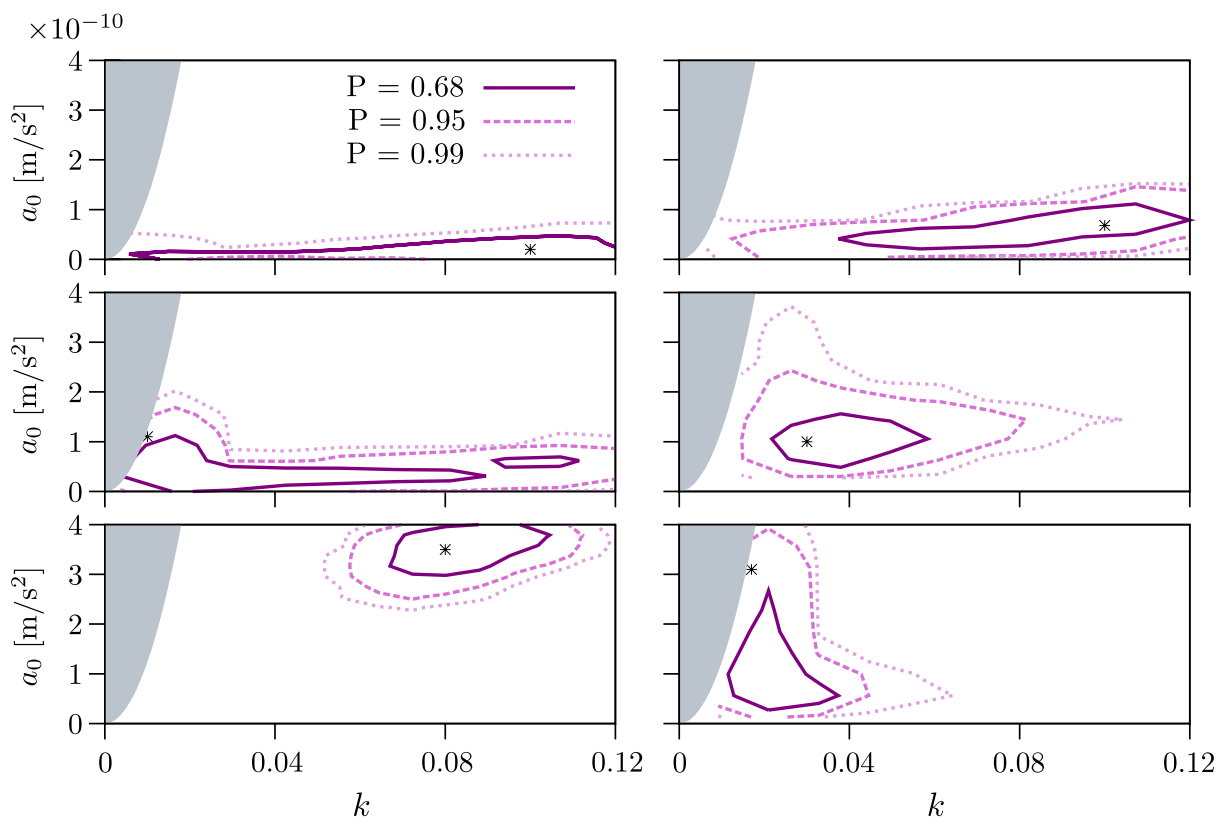

FIG. 17 (color online). Same as Fig. 16 but for the requirements noise model. 
TABLE III. Average values of the standard deviations $\overline{\Delta k}$ and $\overline{\Delta a_{0}}$ of the one-dimensional posteriors of the parameters. The values are given for the six points in the $\left(k, a_{0}\right)$ parameter space where the true signal injections were made. The averages are determined from 200 different noise realizations (using the current best estimate noise) and posterior estimates truncated by our priors are artificially reduced.

\begin{tabular}{|c|c|c|c|c|c|}
\hline \multirow[b]{2}{*}{$\underline{k}$} & \multirow[b]{2}{*}{$a_{0}\left[10^{-10} \mathrm{~m} / \mathrm{s}^{2}\right]$} & \multicolumn{2}{|c|}{ Current best estimate } & \multicolumn{2}{|c|}{ Requirements noise } \\
\hline & & $\overline{\Delta k}$ & $\overline{\Delta a_{0}}\left[10^{-10} \mathrm{~m} / \mathrm{s}^{2}\right]$ & $\overline{\Delta k}$ & $\overline{\Delta a_{0}}\left[10^{-10} \mathrm{~m} / \mathrm{s}^{2}\right]$ \\
\hline 0.030 & 1.00 & 0.00203 & 0.096 & 0.0121 & 0.687 \\
\hline 0.080 & 3.50 & 0.00306 & 0.117 & 0.0125 & 0.352 \\
\hline 0.010 & 1.10 & 0.00087 & 0.225 & 0.0295 & 0.515 \\
\hline 0.017 & 3.10 & 0.00066 & 0.422 & 0.0066 & 0.907 \\
\hline 0.100 & 0.20 & 0.03053 & 0.084 & 0.0345 & 0.173 \\
\hline 0.100 & 0.68 & 0.01838 & 0.137 & 0.0295 & 0.268 \\
\hline
\end{tabular}

relatively small uncertainties. For lower SNRs, however, the error on $k$ is much larger than one on $a_{0}$. In some cases the error on $k$ is limited only by the range of the parameter prior. This scenario will be considered in more detail in Sec. V B, which is dedicated to the case of noise-only simulated data.

Using Eqs. (36a) and (36b) we determine the marginal distributions for the parameters $k$ and $a_{0}$ and their expected values. These marginalized posterior distributions allow us to identify three types of results within our six signal simulations. As shown in Figs. 18 and 19, for the first type of result the joint posterior distribution is narrow and well localized, especially for the current best estimate noise. In this scenario the marginal distributions of both $k$ and $a_{0}$ can be estimated relatively well. Results for the second case can be found in Figs. 20 and 21. This time the posterior is near the boundary of the prior established in Sec. IV B 2. The uncertainty on $a_{0}$ is much broader than the one on $k$. Finally, Figs. 22 and 23 show the third kind of result: the marginalized distribution for $k$ is very broad and is determined by the range that was imposed on it as a prior. In this low SNR regime, it will be hard to make estimates for $k$.

\section{B. The no signal injection case}

No deviations from Newtonian gravity potential have been observed so far in the Solar System. Hence, this is a particularly important case for our analysis and corresponds to a data set containing no signal. We consider this case as a likely outcome of the experiment and wish to assess the impact that a measurement of data with no signal would have on the theory parameter space, i.e. which observationbased restrictions can be placed on the $\left(k, a_{0}\right)$ space.

In Figs. 22 and 23, we already saw the shape of the posterior distribution in the case of low SNRs. We would expect to have somewhat similar results for the case of a noise-only data model, i.e. when we set $\tilde{h}\left(f_{j}, \lambda_{0}^{m}, \lambda_{0}^{t}\right)=0$ in Eq. (15). On the basis of the theory proposed in [20], we place the Newtonian limit of the theory at $a_{0}=0$, thus setting the gravity stress tensor to be equal to the Newtonian stress tensor for all templates on the $k$ axis.
We perform 200 simulations, each with a different noise realization, for both the current best estimate and requirements noise models. We determine $68 \%, 95 \%$, and $99 \%$ confidence interval for both of them. To visualize the restriction on the parameter space that follows, we chose a representative noise realization. The results in Figs. 24 and 25 show uncertainty on the determination of the parameter $k$, meaning that a null measurement would not help us constrain $k$ at all, whereas $a_{0}$ would be tightly bounded. We note, however, that the point in the parameter space for the standard choice of parameters $k=0.03$ and $a_{0}=$ $10^{-10} \mathrm{~m} / \mathrm{s}^{2}$ would be ruled out. The average error on the marginalized posterior distribution of $a_{0}$ for the current best noise estimate is $\overline{\Delta a_{0}}=0.055 \times 10^{-10} \mathrm{~m} / \mathrm{s}^{2}$, while for the requirements noise it is $\overline{\Delta a_{0}}=0.154 \times 10^{-10} \mathrm{~m} / \mathrm{s}^{2}$.

\section{Model selection}

We now follow Eq. (48) and compute the Bayes factor ${ }^{3}$ between our two candidate models $\mathcal{S}$ and $\mathcal{N}$ using the signals calculated for the sets of parameters listed in Table II. This gives a measure of the signal detectability in noise, depending on the combination of the theory parameters $\lambda_{0}^{t}=\left\{k, a_{0}\right\}$, allowing us to quantify the confidence in one model relative to the other on the basis of the outcome of the experiment. As discussed in Sec. IV $\mathrm{E}$, the $\mathcal{S}$ hypothesis assumes that the data is the sum of noise and a signal that depends on $k$ and $a_{0}$, while the $\mathcal{N}$ hypothesis assumes it to be noise only and to have no parameter dependencies. As indicated in Eq. (49), the $\mathcal{S}$ hypothesis requires us to integrate the joint probability $p\left(\{\tilde{x}\}, k, a_{0}\right)$ over the parameter space of the signal $\left(k, a_{0}\right)$, whereas the evidence for the noise-only model is simply given by the likelihood in Eq. (50).

In reality, we will have a single measurement yielding one value for the Bayes factor which itself is a random variable subject to variations between noise realizations. By performing an analysis of the artificial data, however, we can study the distribution of the Bayes factor and therefore

\footnotetext{
${ }^{3}$ We remind the reader that we set the prior model odds to unity.
} 


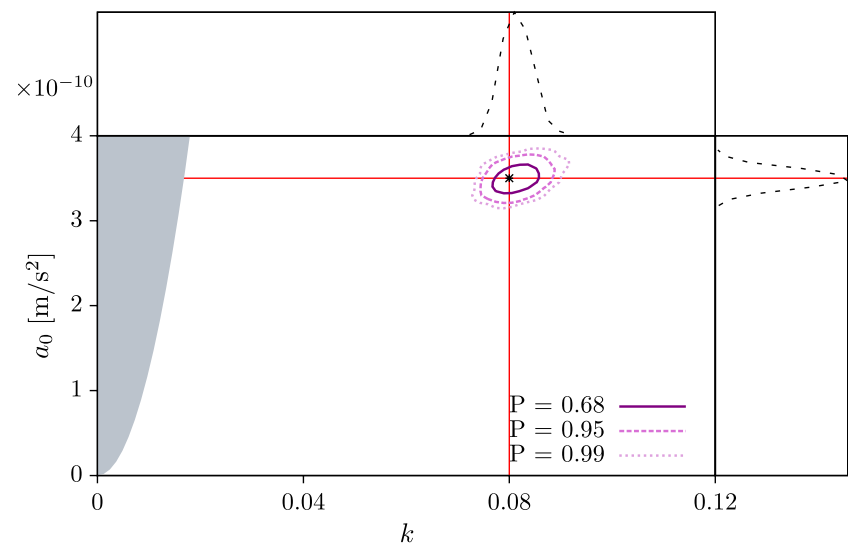

FIG. 18 (color online). Posterior probability distributions and marginalized posterior distributions for the current best noise estimate for the parameters of the injected signal at $k=0.08$ and $a_{0}=3.5 \times 10^{-10} \mathrm{~m} / \mathrm{s}^{2}$. The red lines indicate the true values at which the simulated signal was injected.

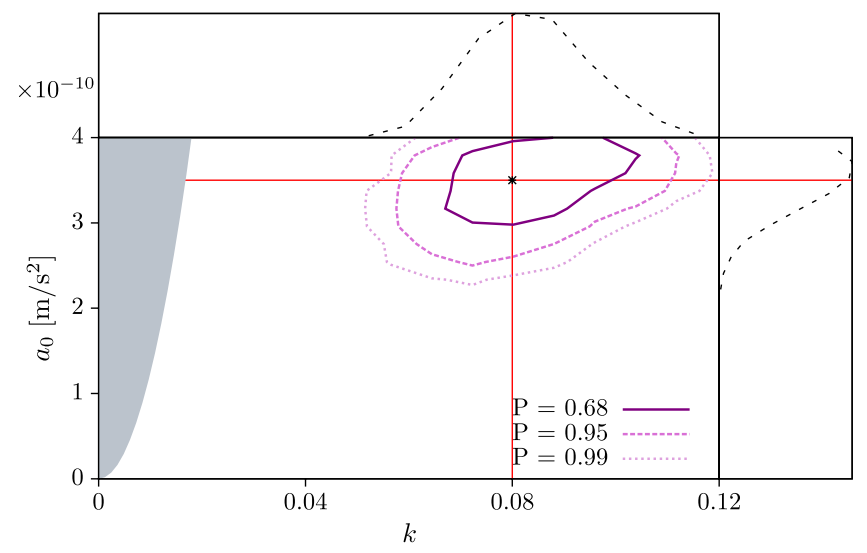

FIG. 19 (color online). Posterior probability and marginalized posterior distributions for requirements noise for the parameters of the injected signal at $k=0.08$ and $a_{0}=3.5 \times 10^{-10} \mathrm{~m} / \mathrm{s}^{2}$. The red lines indicate the true values at which the simulated signal was injected.

understand the interpretation of a single value measurement. For the model selection we analyzed the same data as for the parameter estimation. The Bayes factor distributions dependence upon the theory parameters is found in Fig. 26 for the current best estimate noise model and in Fig. 27 for the requirements noise. We show the logarithms of the Bayes factor estimates at the seven representative points in the parameter space collected in Table II. In five cases out of seven the Bayes factor logarithms all have positive values: this means that the $\mathcal{S}$ hypothesis will be strongly favored over the $\mathcal{N}$ hypothesis. On the other hand, negative logarithms of the Bayes factor imply that the noise-only model $\mathcal{N}$ is favored. This occurs in two cases out of seven. One of these is the noise-only $\left(k=0, a_{0}=0 \mathrm{~m} / \mathrm{s}^{2}\right)$ point, where the data only contains noise: this behavior is

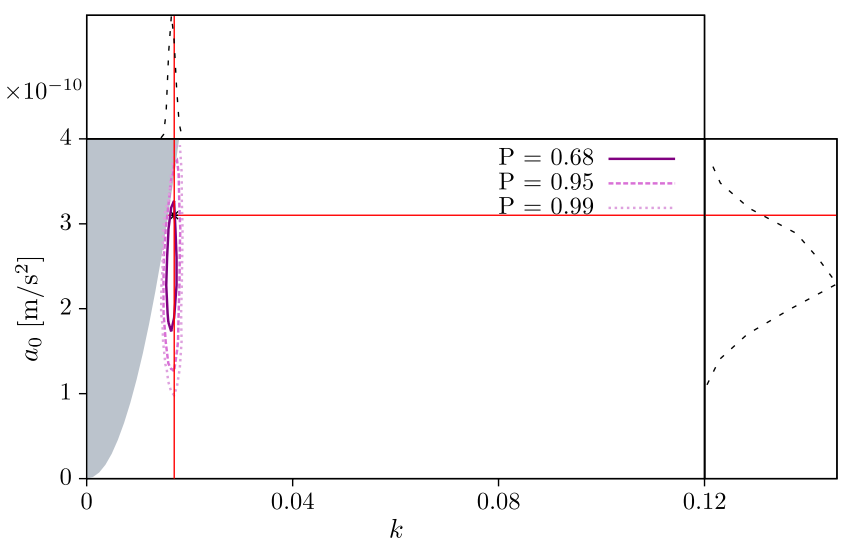

FIG. 20 (color online). Posterior probability distributions and marginalized posterior distributions for the current best noise estimate for the parameters of the injected signal $k=0.017$ and $a_{0}=3.1 \times 10^{-10} \mathrm{~m} / \mathrm{s}^{2}$. The red lines indicate the true values at which the simulated signal was injected.

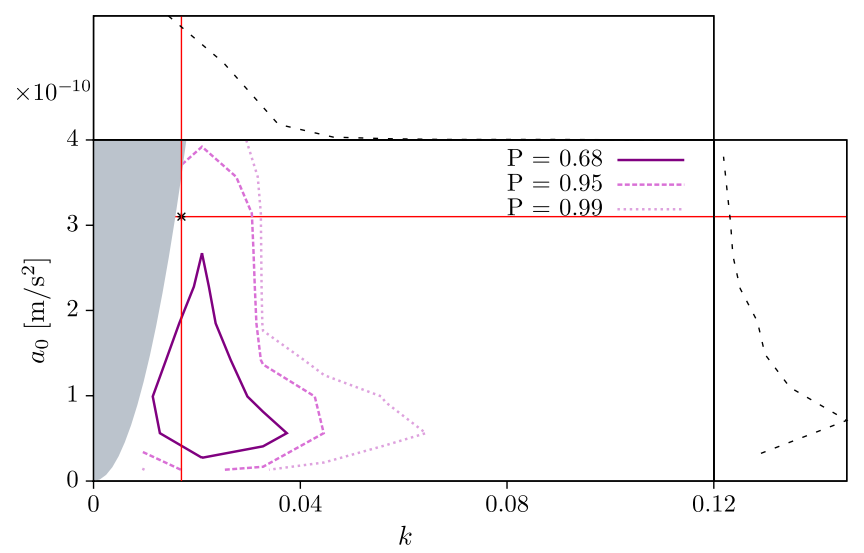

FIG. 21 (color online). Same as Fig. 20 but for the requirements noise.

therefore expected. The second point is at $(k=0.1$, $\left.a_{0}=0.2 \times 10^{-10} \mathrm{~m} / \mathrm{s}^{2}\right)$. In this case, noise and signal are mixed, but a rejection of the $\mathcal{S}$ hypothesis is likely.

The analysis just discussed shows a rigorous way of determining the detectability of a signal. While we solely considered a noise-only model and a signal model of MONDian inspiration, we note that our analysis can be extended to include other models, as, for example, models with non-Gaussian noise or ones incorporating glitches that could resemble the signal. In addition we can probe whether the data will be best described by one theory or another when it exhibits a deviation from the Newtonian background.

\section{Detector noise artifacts}

So far we analyzed the simulated LPF data with noise taken to be Gaussian and ASD defined by the theoretical 


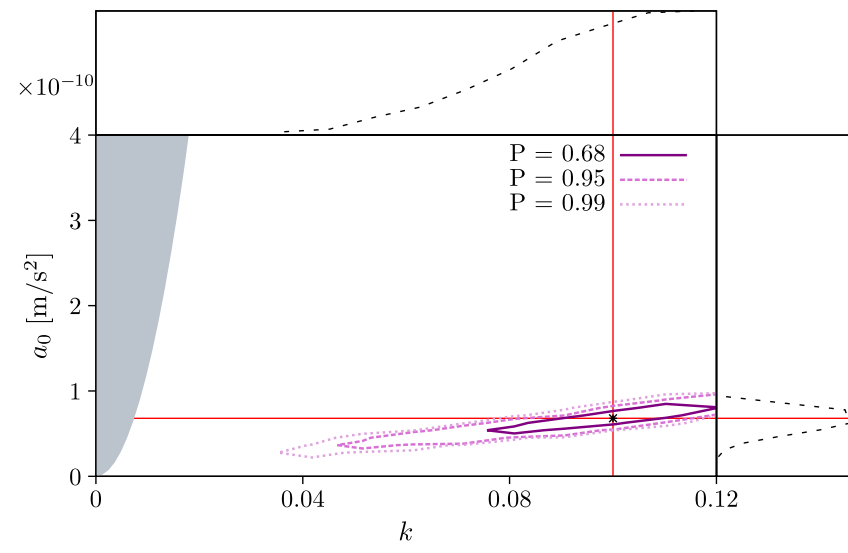

FIG. 22 (color online). Posterior probability distributions and marginalized posterior distributions for the current best noise estimate for parameters of the signal modelled for $k=0.1$ and $a_{0}=0.68 \times 10^{-10} \mathrm{~m} / \mathrm{s}^{2}$. The red lines indicate the true values at which the simulated signal was injected.

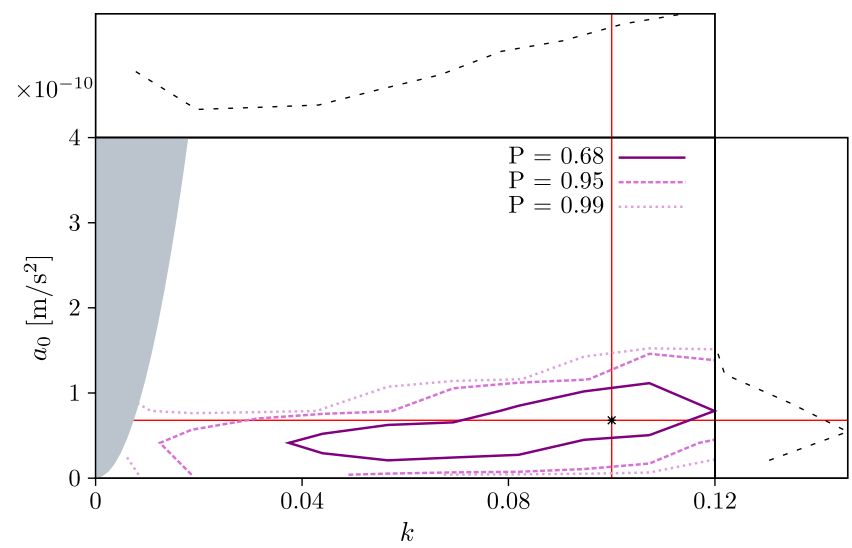

FIG. 23 (color online). Same as Fig. 22 but for the requirements noise.

amplitude spectral density of LPF. In reality, however, nonGaussian glitches might appear in the noise as shown in the measurement of the differential displacements from the test campaigns for $\mathrm{LPF}^{4}$ [33-35]. We now examine the response of our data analysis framework to glitches by performing parameter estimation and model selection on the OSTT data. We keep working in the TeVeS $\left(k, a_{0}\right)$ parameter space and use the signal templates produced within this theory.

\footnotetext{
${ }^{4}$ The LPF spacecraft is already being prepared for launch and is undergoing several instrumental tests. To assess the impact of noise artifacts, we took the data available from the LPF On-Station Thermal Tests (OSTT) performed by Astrium Ltd., Astrium Satellite GmbH [Astrium Deutschland (ASD)] extensively testing the end-to-end performance of the Optical Metrology System (OMS). However, we would like to point out that the noise artifacts might have been artificially caused by the test environment.
}

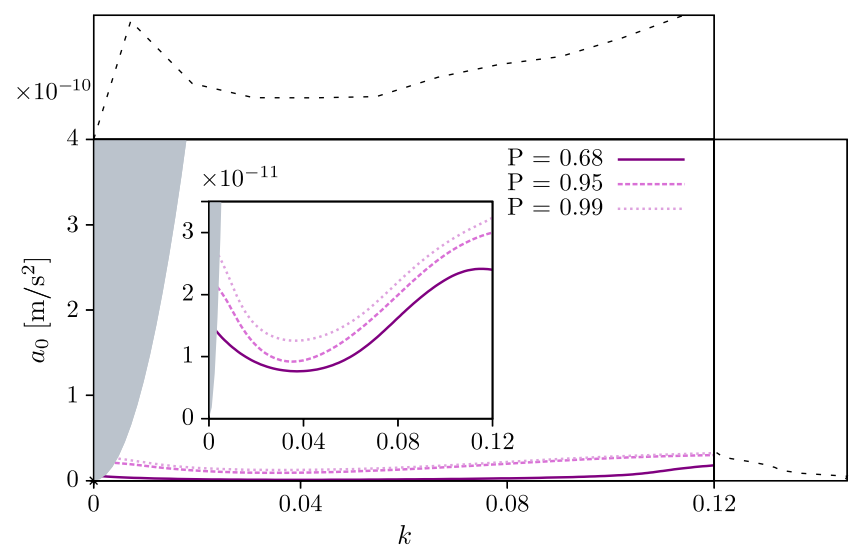

FIG. 24 (color online). Posterior probability density for the current best estimate noise realization in the case of no signal injection, i.e. $\tilde{h}\left(f_{j}, \lambda_{0}^{m}, \lambda_{0}^{t}\right)=0$.

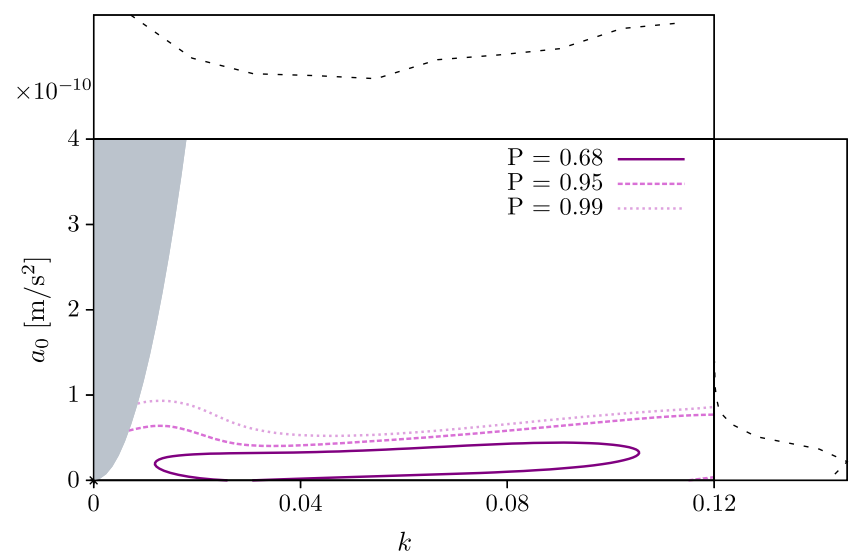

FIG. 25 (color online). Same as Fig. 24 but for the requirements noise.

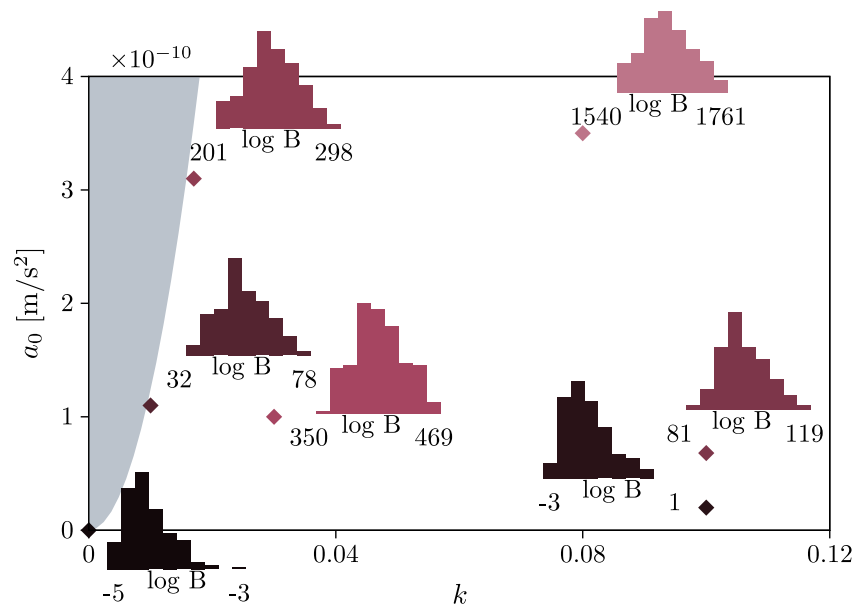

FIG. 26 (color online). Histograms of the logarithms of the Bayes factor $\log B$ for the 200 noise realizations (current best estimate) at the seven representative points in the parameter space, where the signals were injected. These points are listed in Table II. 


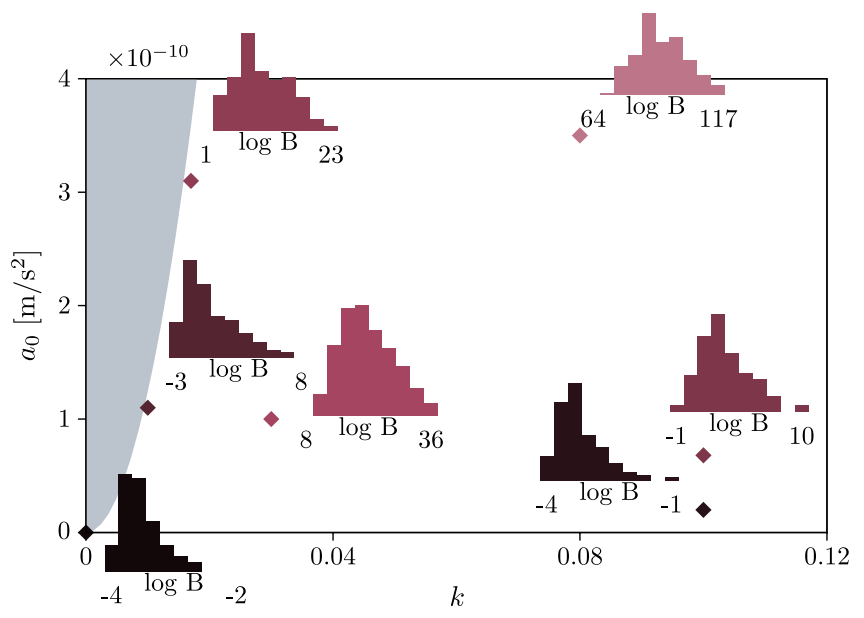

FIG. 27 (color online). Same as Fig. 26 but for the requirements noise.

We shift the test campaign data so that a glitch occurs at the expected signal arrival time, as shown in Fig. 28. We then estimate the posterior probability distribution for $k$ and $a_{0}$ for this data set. Results are presented in Fig. 29. The posterior probability peaks at $\left(k=0.12, a_{0}=\right.$ $\left.1.34 \times 10^{-10} \mathrm{~m} / \mathrm{s}^{2}\right)$. The standard deviations for the two parameters are given by $\Delta k=0.001$ and $\Delta a_{0}=$ $0.07 \times 10^{-10} \mathrm{~m} / \mathrm{s}^{2}$, respectively. The recovered parameter values are in the parameter space region that is inconsistent with the noise-only model. Additionally, the estimated value of the parameter $k$ is on the boundary of the parameter range defined by the parameter priors.

The logarithm of the Bayes factor is $\log p(\mathcal{S} \mid\{\tilde{x}\}, I) /$ $p(\mathcal{N} \mid\{\tilde{x}\}, I)=199$, so that the $\mathcal{S}$ hypothesis is prioritized over the $\mathcal{N}$ one. This can happen if the characteristic frequency of the glitch is similar to the characteristic frequency of the signal and highlights that, in order to achieve confident signal detection, we must introduce more realistic noise models. In particular, these should describe non-Gaussianities in the noise, such as glitches. With such

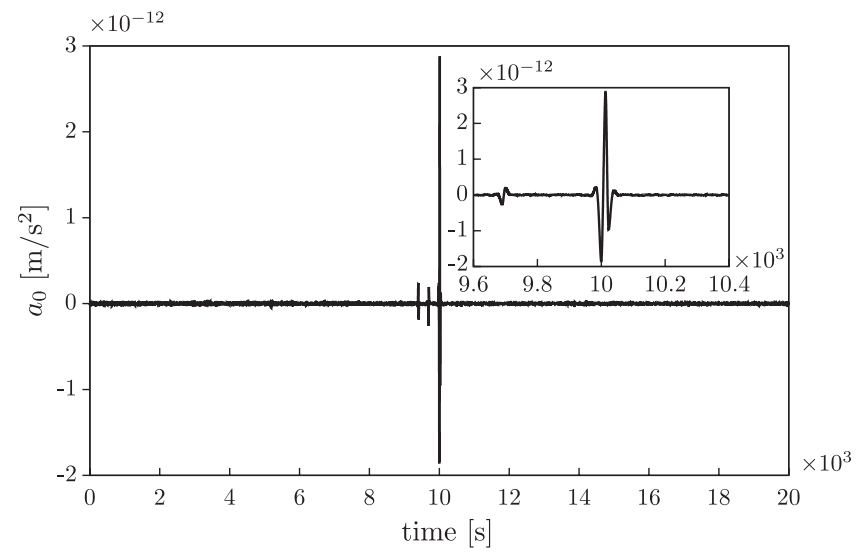

FIG. 28. Data from the test campaign with a glitch of unknown origin bandpass filtered in the sensitive frequency band of LPF.

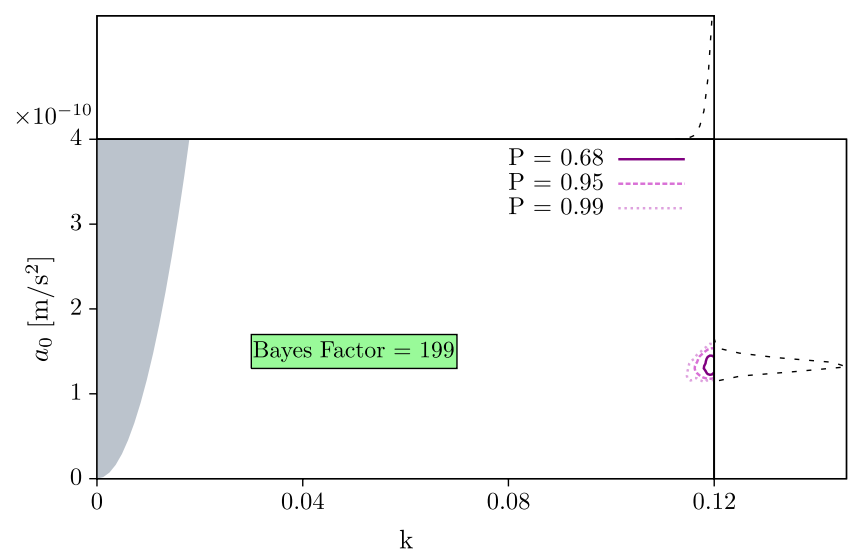

FIG. 29 (color online). Resulting posterior probability density for the parameter estimation in case of the realistic data of Fig. 28.

noise models it would be possible to extend the model selection described in Sec. IV E and distinguish between noise artifacts and authentic signals. The question of the nonstationarities and glitches in the data is particularly important in the setup of this experiment because our measurement relies on one or two repetitions at the most (one or two SP fly-by's). Multiple SP fly-by's can significantly increase our confidence in signal detection against glitches in the data. However, distinguishing between noise glitches and signal, and characterizing glitches are very important topics that will need further investigation.

\section{CONCLUSIONS AND FUTURE WORK}

In this paper we developed a data analysis approach to test alternative theories of gravity with LPF. As shown in Eq. (4), the gravitational stress tensor affects the relative acceleration between the two test masses onboard the spacecraft. The tidal field can be sampled by LPF, allowing us to measure its (dis)agreement with the Newtonian tidal field. The time series that an LPF measurement will provide depends on the trajectory of the spacecraft and on the orientation of its sensitive axis via the seven mission parameters listed in Eq. (14). The data analysis framework we built will allow for quantitative statements on measuring the tidal field and posing constraints on alternative theories of gravity.

Testing our data analysis approach required picking a theory of gravity that predicts deviations from the Newtonian tidal stresses within the Solar System, where LPF will fly. As discussed in Sec. IV B, we considered the example of the $\mathrm{TeV} e \mathrm{~S}$ theory. This choice is convenient as we are able to calculate signal templates from it. In the regime of our interest, the signal measured by LPF depends on two theory parameters only, namely, a dimensionless coupling constant $k$ and a characteristic acceleration $a_{0}$. Having picked an alternative theory of gravity, we were able to quantify how the signal is influenced by variations of each of the mission parameters. We concluded that, 
within the errors on the measurement of the position of the spacecraft, the variations of the signal will be negligible. This is a crucial result as it allows us to fix the values of the mission parameters when building signal templates in order to carry out a Bayesian analysis of the theory parameter estimation and a model selection. However, the impact of possible correlations between the mission and the theory parameters on the conclusions drawn so far in our study was not assessed. Nevertheless, we expect this correlation to be insignificant and leave this investigation for the future work.

The results of our Bayesian analysis are presented in Sec. $\mathrm{V}$ in the form of posterior distributions for the two theory parameters that determine the signal. These are obtained by considering 200 different noise realizations. Some combinations of the parameters (point 1 and point 2 in Table II) yield a sharp and narrow joint posterior distribution, indicating that it will be possible to estimate the theory parameters with high precision in case of high SNRs. For weak signals with low SNRs (point 5 and point 6 in Table II) the parameter $k$ can only be poorly estimated from the posterior probability. The results for the current best estimate of the noise systematically exhibit better parameter estimation and better distinction between the noise and the signal hypotheses (see Figs. 26 and 27) than the requirements noise. As the former model was built upon the estimates of the noise from the flight hardware test campaigns (see Sec. II C), it is a good approximation of the noise during flight.

We also considered the special case in which the data consists of noise only, i.e. a modified gravity signal is absent. This is a very important case as it is a priori the most likely possible outcome of the experiment. In this scenario, the parameter space outside the confidence area of the posterior distribution can be ruled out. In the case of no signal injection, we obtained an average error on the determination of $a_{0}$ which is $\overline{\Delta a_{0}}=0.055 \times 10^{-10} \mathrm{~m} / \mathrm{s}^{2}$ for the current best estimate noise model and $\overline{\Delta a_{0}}=0.154 \times 10^{-10} \mathrm{~m} / \mathrm{s}^{2}$ for the requirements noise. This rules out most values of $a_{0}$ except those that are close to $0 \mathrm{~m} / \mathrm{s}^{2}$. At the same time, there is a complete uncertainty on $k$, which means that we will not be able to draw any conclusions on this parameter in case of no signal detection.

In order to distinguish between signal detection and no signal detection, we used the Bayesian approach to model selection. We limited the choice to two models: one is the sum of noise and signal (signal hypotheses), while the other consists of noise only (noise hypothesis). We computed the ratio of the probabilities for these two hypotheses given the data and based on this number drew a conclusion on which model is preferable. We estimated the expectation for a signal in the artificial data by calculating Bayes factors for 200 different noise realization for several points in the parameter space listed in Table II. On the basis of these estimates, we were able to allocate areas in the parameter space where the signal hypothesis could be strongly prioritized over the noise hypothesis and areas where even in the presence of a signal a confident statement on its detection cannot be made. Notice that for a single fly-by the experiment will provide us only with a single measured data set and a single deduced Bayes factor. The estimates of the Bayes factors for the artificial data gives a way to compare the single Bayes factor estimated from the real data to the expected values and judge the outcome of the experiment on the basis of this comparison.

Finally, we studied the data from one of the test campaigns for LPF. The importance of this study lies in the fact that in reality the noise may have glitches and non-Gaussianities (see Fig. 28). When applied to this data, our Bayesian model selection can prefer the signal hypothesis over the noise hypothesis because neither of them describes the data with the glitch correctly. In order to adequately address the problem of glitches, a separate model to be fed to the Bayesian hypothesis selection approach must be developed.

In our analysis we investigated the influence of the parameters $k$ and $a_{0}$ on the template, but we kept the interpolating function fixed. As the interpolating function is heuristically designed on the basis of astrophysical observations, it is not a smoothly varying parameter but a point model. In a future work, we would like to apply the data analysis framework we built to study a generalized, phenomenological model of the interpolating function that uses a finite set of parameters. This would allow us to assess different theories that have MOND as their nonrelativistic limit. Ultimately, the more general goal is to consider other theories that yield a phenomenology detectable with LPF and to be able to perform a model selection among different models of gravity.

The significant issue left out of the scope of this paper is the influence of the mission design and the mission timeline on the experiment. We leave it to future work to study the influence of the accuracy of the acceleration recovery from the measurement of the displacement on the parameter estimation. Finally, the question of how much data before and after the SP fly-by needs to be gathered to perform an accurate estimation of the acceleration and to assess the possible non-Gaussianities in the noise is also left for future work.

\section{ACKNOWLEDGMENTS}

The authors would like to thank B. Sathyaprakash, Christian Trenkel, Stephen Kemble, Badri Krishnan, Reinhard Prix, Ali Mozaffari, Luc Blanchet, Paul McNamara, Gilles Esposito-Farèse, and Philippe Jetzer for useful discussions, and Carsten Aulbert and Henning Fehrmann for their comprehensive help. The numerical calculations were performed on the Datura cluster at the Max Planck Institute for Gravitational Physics in Golm and on the Atlas Cluster at the Max Planck Institute for Gravitational Physics in Hannover. The code used to solve the nonrelativistic $\mathrm{TeV} e \mathrm{~S}$ equations was kindly provided 
by the Imperial College London. The authors would like to especially thank João Magueijo and Neil Bevis for providing the code. The authors acknowledge Astrium Satellite $\mathrm{GmbH}$ (ASD), Germany for providing the OSTT data from the LPF test campaign. F. P. acknowledges support from STFC Grant ST/L000342/1 and DFG Grant SFB/ Transregio 7.

\section{APPENDIX A: TeVeS}

$\mathrm{T} e \mathrm{~V} e \mathrm{~S}$ was the first consistent relativistic theory of gravity reducing to MOND in the nonrelativistic limit. It is built upon a nondynamical gravitational scalar field $\sigma$ and three dynamical gravitational fields, namely, the Einstein metric tensor $g_{\alpha \beta}$, a timelike 4-vector field $\mathfrak{U}^{\beta}$, and a scalar field $\phi$. Accordingly, it was dubbed tensor-vector-scalar theory. The physical metric may be obtained from the dynamical fields via the relation $\tilde{g}_{\alpha \beta}=e^{-2 \phi} g_{\alpha \beta}-$ $2 \mathfrak{U}_{\alpha} \mathfrak{U}_{\beta} \sinh (2 \phi)$, where and $\mathfrak{U}_{\alpha}=g_{\alpha \beta} \mathfrak{U}^{\beta}$.

Within this theory, the total action takes the form

$$
S=S_{g}+S_{v}+S_{s}+S_{m},
$$

where $S_{g}$ is the Einstein-Hilbert action for the metric tensor, $S_{v}$ is the action governing the timelike vector field, $S_{s}$ is the action for the dynamical and the nondynamical scalar fields, and $S_{m}$ is the action for the matter fields. The equation for the dynamical gravitational scalar field may be derived from

$S_{s}=-\frac{1}{2} \int\left[\sigma^{2} h^{\alpha \beta} \phi_{, \alpha} \phi_{, \beta}+\frac{1}{2} G l^{-2} \sigma^{4} F\left(k G \sigma^{2}\right)\right] \sqrt{-g} \mathrm{~d}^{4} x$,

where $g=\operatorname{det}\left(g_{\alpha \beta}\right), h^{\alpha \beta} \equiv g^{\alpha \beta}-\mathfrak{U}^{\alpha} \mathfrak{U}^{\beta}, G$ is the gravitational constant, $k$ is a dimensionless constant, $l$ is a constant length, and $F$ is a free dimensionless function. Varying $S_{s}$ with respect to the two scalar fields and using the equation for $\sigma$ yields [20]

$\left[\mu\left(k l^{2} h^{\mu \nu} \phi_{, \mu} \phi_{, \nu}\right) h^{\alpha \beta} \phi_{, \alpha}\right]_{; \beta}=k G\left[g^{\alpha \beta}+\left(1+e^{-4 \phi}\right) \mathfrak{U}^{\alpha} \mathfrak{U}^{\beta}\right] \tilde{T}_{\alpha \beta}$,

where $\tilde{T}_{\alpha \beta}$ is the physical energy-momentum tensor, i.e. built upon the physical metric $\tilde{g}_{\alpha \beta}$, and the function $\mu(y)$ obeys

$$
-\mu F(\mu)-\frac{1}{2} \mu^{2} \frac{\mathrm{d} F(\mu)}{\mathrm{d} \mu}=y .
$$

\section{APPENDIX B: NEWTONIAN STRESS TENSOR}

The expression of the Newtonian potential $\Phi_{\mathrm{N}}$ for the Sun-Earth two-body system is

$$
\Phi_{\mathrm{N}}=-G\left[M_{\mathrm{e}} \frac{r_{0}-d_{\mathrm{e}}}{d_{\mathrm{e}} r_{0}}+M_{\mathrm{s}} \frac{r_{\mathrm{se}}-r_{0}-d_{\mathrm{s}}}{d_{\mathrm{s}}\left(r_{\mathrm{se}}-r_{0}\right)}\right],
$$

where $G$ is Newton's gravitational constant, $M_{\mathrm{S}}\left(M_{e}\right)$ is the mass of the Sun (Earth), $r_{\text {se }}$ is the Sun-Earth separation,

$$
r_{0}=\frac{r_{\mathrm{se}} \sqrt{M_{\mathrm{e}} / M_{\mathrm{s}}}}{\sqrt{M_{\mathrm{e}} / M_{\mathrm{s}}}+1}=\frac{r_{\mathrm{se}}}{\sqrt{M_{\mathrm{s}} / M_{\mathrm{e}}}+1}
$$

is the distance from the Earth to the SP, and $d_{\mathrm{S}}\left(d_{\mathrm{e}}\right)$ is the distance from the point where the potential is calculated to the Sun (Earth) respectively, i.e.

$$
\begin{gathered}
d_{\mathrm{e}}=\sqrt{\left(x_{1}+r_{0}\right)^{2}+x_{2}^{2}+x_{3}^{2}}, \\
d_{\mathrm{s}}=\sqrt{\left(\left(r_{\mathrm{se}}-r_{0}\right)-x_{1}\right)^{2}+x_{2}^{2}+x_{3}^{2}} .
\end{gathered}
$$

The gradient of the Newtonian potential is therefore

$$
\frac{\partial \Phi_{\mathrm{N}}}{\partial x_{i}}=\frac{G M_{\mathrm{e}}\left[x_{i}-r_{0} c_{i}\right]}{d_{\mathrm{e}}^{3}}+\frac{G M_{\mathrm{s}}\left[x_{i}-\left(r_{\mathrm{se}}-r_{0}\right) c_{i}\right]}{d_{\mathrm{s}}^{3}},
$$

where $\hat{\boldsymbol{e}}_{x_{i}}(i=1 \ldots 3)$ is the orthonormal unit vectors set of the reference system and $c_{i}=\hat{\boldsymbol{e}}_{x_{1}} \cdot \hat{\boldsymbol{e}}_{x_{i}}$. The Newtonian stress tensor reads

$$
\begin{aligned}
\frac{\partial^{2} \Phi_{\mathrm{N}}}{\partial x_{i}^{2}}= & G M_{\mathrm{e}}\left\{\frac{1}{d_{\mathrm{e}}^{3}}-\frac{3\left[x_{i}+r_{0} c_{i}\right]^{2}}{d_{\mathrm{e}}^{5}}\right\} \\
& +G M_{\mathrm{s}}\left\{\frac{1}{d_{\mathrm{s}}^{3}}-\frac{3\left[x_{i}-\left(r_{\mathrm{se}}-r_{0}\right) c_{i}\right]^{2}}{d_{\mathrm{s}}^{5}}\right\}, \\
\left.\frac{\partial^{2} \Phi_{\mathrm{N}}}{\partial x_{i} \partial x_{j}}\right|_{i \neq j}= & -\frac{3 G M_{\mathrm{e}}}{d_{\mathrm{e}}^{5}}\left[x_{i}+r_{0} c_{i}\right]\left[x_{j}+r_{0} c_{j}\right] \\
& -\frac{3 G M_{\mathrm{s}}}{d_{\mathrm{s}}^{5}}\left[x_{i}-\left(r_{\mathrm{se}}-r_{0}\right) c_{i}\right]\left[x_{j}-\left(r_{\mathrm{se}}-r_{0}\right) c_{j}\right] .
\end{aligned}
$$


[1] F. Antonucci et al., Classical Quantum Gravity 29, 124014 (2012).

[2] P. Amaro Seoane et al., arXiv:1305.5720.

[3] ESA, "L3 Science Theme", http://sci.esa.int/jump.cfm? oid $=53259$.

[4] G. D. Racca and P. W. McNamara, Space Sci. Rev. 151, 159, (2010).

[5] J. Bekenstein and J. Magueijo, Phys. Rev. D 73, 103513 (2006).

[6] C. Trenkel and S. Kemble, J. Phys. Conf. Ser. 154, 012002 (2009).

[7] J. H. Oort, Bull. Astron. Inst. Neth. 6, 249 (1932).

[8] V. C. Rubin and W. K. Ford, Jr., Astrophys. J. 159, 379 (1970).

[9] J. Ostriker and P. Peebles, Astrophys. J. 186, 467 (1973).

[10] A. Bosma, Astron. J. 86, 1825 (1981).

[11] V. C. Rubin, W. K. Ford, Jr., N. Thonnard, and D. Burstein, Astrophys. J. 261, 439 (1982).

[12] F. Zwicky, Gen. Relativ. Gravit. 41, 207 (2009).

[13] M. Milgrom, Astrophys. J. 270, 365 (1983).

[14] N. Bevis, J. Magueijo, C. Trenkel, and S. Kemble, Classical Quantum Gravity 27, 215014 (2010).

[15] P. C. C. Freire, N. Wex, G. Esposito-Farèse, J. P. W. Verbiest, M. Bailes, B. A. Jacoby, M. Kramer, I. H. Stairs, J. Antoniadis, and G. H. Janssen, Mon. Not. R. Astron. Soc. 423, 3328 (2012).

[16] G. Esposito-Farèse, in Mass and Motion in General Relativity, edited by L. Blanchet, A. Spallicci, and B. Whiting (Springer, New York, 2011), pp. 461-489.

[17] S. Vitale, Report No. S2-UTN-TN-3053, 2007.

[18] A. Monsky et al., Classical Quantum Gravity 26, 094004 (2009).

[19] C. W. Misner, K. S. Thorne, and J. A. Wheeler, Gravitation (W. H. Freeman, San Francisco, 1973).

[20] J. D. Bekenstein, Phys. Rev. D 70, 083509 (2004).

[21] B. Famaey and S. McGaugh, Living Rev. Relativity 15, 10 (2012).
[22] Computer clusters at the AEI, http://www.aei.mpg.de/ 96456/20_Computer_Clusters.

[23] E. Fabacher, S. Kemble, C. Trenkel, and N. Dunbar, Adv. Space Res. 52, 105 (2013).

[24] C. Trenkel and S. Kemble (private communication).

[25] C. Trenkel, S. Kemble, N. Bevis, and J. Magueijo, Adv. Space Res. 50, 1570 (2012).

[26] S. V. Dhurandhar and B. S. Sathyaprakash, Phys. Rev. D 49, 1707 (1994).

[27] B. S. Sathyaprakash and B. F. Schutz, Living Rev. Relativity 12, 2 (2009).

[28] A. Fienga, J. Laskar, P. Kuchynka, C. Le Poncin-Lafitte, H. Manche, and M. Gastineau, in Proceedings of the International Astronomical Union, edited by S. A. Klioner, P. K. Seidelmann, and M. H. Soffel (Cambridge University Press, Cambridge, England, 2010), Vol. 5, pp. 159-169.

[29] J. Skilling, AIP Conf. Proc. 735, 395 (2004).

[30] D. Sivia and J. Skilling, Data Analysis: A Bayesian Tutorial (Oxford University Press, Oxford, 2006).

[31] J. Skilling, Bayesian Analysis 1, 833 (2006).

[32] F. Feroz, M. P. Hobson, and M. Bridges, Mon. Not. R. Astron. Soc. 398, 1601 (2009).

[33] G. Hechenblaikner and R. Flatscher, in Society of PhotoOptical Instrumentation Engineers (SPIE) Conference Series, edited by B. Bodermann, K. Frenner, and R. M. Silver, SPIE Proceedings (SPIE-International Society for Optical Engineering, Bellingham, WA, 2013), Vol. 8789.

[34] G. Hechenblaikner, R. Gerndt, U. Johann, P. LuetzowWentzky, V. Wand, H. Audley, K. Danzmann, A. Garcia-Marin, G. Heinzel, M. Nofrarias, and F. Steier, Appl. Opt. 49, 5665 (2010).

[35] R. Flatscher, R. Gerndt, K. Rogalla, N. Gradmann, and D. Kolbe, Test Report on OMS Performance Parameter Characterization During OSTT, Technical Report \{ Astrium Ltd., Astrium Satellite GmbH [Astrium Deutschland (ASD)], 2013\}. 